\title{
离散抽样 Gamma-OU 过程的参数估计*
}

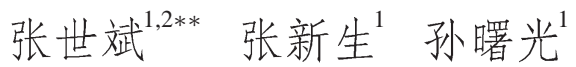 \\ (1. 复旦大学管理学院统计学系, 上海 200433; 2. 上海海事大学数学系, 上海 200135)
}

\begin{abstract}
摘要平稳 Gamma-OU 过程是用于刻画金融资产波动的一类重要模型. 本文 主要考虑基于离散观察的 Gamma-OU 过程的参数估计. 文中给出了强度参数 $\lambda$ 的估计量及其收玫性, 模拟显示这一估计是相当准确的. 在假设参数 $\lambda$ 已被估 计出来的条件下, 又研究了形状参数 $c$ 和尺度参数 $\alpha$ 的最大似然估计, 其中关于 这两个参数的似然函数是难于计算的. 通过 Gaver-Stehfest 算法, 我们构造了一 个似然函数的具体估计序列, 它收玫于真实 (但未知) 的似然函数. 最大化这一 序列可以得到收玫于真实最大似然估计的一列估计量, 并且这一估计序列具有 与最大似然估计相同的收玫性. 模拟显示在大多数有关波动率的实际背景下, 我们的方法是非常准确的.
\end{abstract}

关键词 Gamma-OU 过程 转移函数 Lévy 过程 Lévy 密度 随机波动 驱动 Lévy 过程 Laplace 变换 最大似然估计

\section{1 引言}

在金融和计量经济中, $\mathrm{OU}$ 型非 Gauss 过程得以强有力的应用. 本文主要 研究一类连续时间的随机波动模型, 该模型由 Barndorff-Nielsen 和 Shephard ${ }^{[1]}$ 提 出, 用于刻画金融资产的波动, 称为 Gamma-OU 过程. 在本文中, 随机波动 $\sigma^{2}(t)$ 满足随机微分方程

$$
\mathrm{d} \sigma^{2}(t)=-\lambda \sigma^{2}(t) \mathrm{d} t+\mathrm{d} z(\lambda t),
$$

其中 $z(t)$ 为具有正增量的 Lévy 过程, 称为驱动 Lévy 过程. 与众不同的时间选择 $\mathrm{d} z(\lambda t)$ 是故意的, 这样选择之后, 无论 $\lambda$ 取何值, $\sigma^{2}(t)$ 的边际分布都是与其无 关的 Gamma 分布, 因此称 $\sigma^{2}(t)$ 为 Gamma-OU 过程 ${ }^{[2]}$. 
虽然我们的模型是连续时间的, 但是实际数据总是关于时间离散抽取的. 最 近估计扩散过程参数的一些计量经济学方法得以发展, 在这些方法中, 无需连续 记录观测值, 其中最大似然方法备受青棟. 对于离散时间抽样情形, 目前可获得 的计算似然函数的方法, 或者通过计算 Fokker-Planck-Kolmogorov 偏微分方程的 数值解 ${ }^{[3]}$, 或者通过模拟很多抽样精细的样本轨道 ${ }^{[4,5]}$. 上述任何一种方法都没 有产生可以在参数空间内最大化的闭形式 (closed-form), Ait-Sahalia 的工作 ${ }^{[6]}$ 改 变了这一现状, 与其类似的工作还有文献 [7], 他们的方法都是通过转移密度的 Hermite 展开来估计似然函数. 对于 Lévy 驱动 OU 过程的情形, Barndorff-Nielsen 和 Shephard ${ }^{[8]}$ 采用实现波动率 (realized volatility), 即他们利用一天中的高频数据直接 估计积分波动的矩来估计参数. 对于 Gamma-OU 过程的情形, Griffin 和 Steel ${ }^{1)}$ 提 出并发展了 Bayes 方法, 他们采用建立在模拟高频观测数据增加基础上的 Markov 链 Monte Carlo 方法 (MCMC)； MCMC 方法也被 Roberts 和 Papaspiliopoulos ${ }^{[9]}$ 所 采用, 他们的方法中含有根据一个合适的标点 Poisson 过程表示没有观测到的随 机波动过程的思想. 非参数方法由 Jongbloed 等人 [10] 所采纳.

假设我们信赖由方程 (1.1) 所决定的参数结构, 则最大似然方法是可供选择 的一个较好的方法, 因为它的估计量一般具有较好的性质, 且可避免 MCMC 方 法中大量的模拟工作. 本文估出参数 $\lambda$ 之后, 得到了可以在参数空间上最大化 的闭形式. 通过卷积, 得到了转移密度的形式, 通过 Gaver-Stehfest算法, 得到其 近似算法. 并且最终的对数似然函数及其近似可以通过对起初似然函数做适当 处理得到.

本文假设有离散观测值 $X_{k}=\sigma^{2}\left(t_{k}\right)$, 其中 $\left\{t_{k}=k \Delta, k=0,1, \cdots, n\right\}, \Delta>0$ 是固定的. 通过前面的介绍, 我们主要考虑估计强度参数 $\lambda$ 和估计平稳分布参数 $(c, \alpha)$ 两个问题. 参数 $(c, \alpha)$ 的估计方法是最大似然方法, 其实现通过 Gamma-OU 过程转移密度的近似.

\section{Gamma-OU 过程的转移函数}

\subsection{Gamma-OU 过程的结构}

在我们的模型中, $\sigma^{2}(t)$ 的边际分布为具有形状参数 $c$ 和尺度参数 $\alpha$ 的 Gamma 分布.

$\sigma^{2}(t)$ 的 Lévy 密度为 $u(x)=\frac{c e^{-\alpha x}}{x} \mathbb{I}_{\{x>0\}}$, 其相应的特征函数是

$$
\psi(z)=\exp \left(\int_{0}^{\infty}\left(e^{i z x}-1\right) \frac{c e^{-\alpha x}}{x} \mathrm{~d} x\right)=\left(1-\frac{i z}{\alpha}\right)^{-c} .
$$

因为 $u(x)$ 是可微的, 故可利用 $\sigma^{2}(t)$ 和 $z(1)$ Lèvy 密度之间的关系 ${ }^{[2]}$ 得到 $z(1)$ 的

1) Griffin J E, Steel M F J. Inference with Non-Gaussian Ornstein-Uhlenbeck processes for stochastic volatility, which is provided by Economics Working Paper Archive EconWPA in its series Econometrics with number 0201002, 2005. 
Lévy 密度

$$
w(x)=-u(x)-x u^{\prime}(x)=c \alpha e^{-\alpha x} \mathbb{I}_{\{x>0\}},
$$

其相应的特征函数是

$$
\phi(z)=\exp \left(\int_{0}^{\infty}\left(e^{i z x}-1\right) c \alpha e^{-\alpha x} \mathrm{~d} x\right) .
$$

因此, 不难验证 $z(t)$ 是一复合 Poisson 过程, 且 $z(t)$ 具有表示形式 $z(t)=\sum_{i=1}^{N(t)} Y_{U_{i}}$, 其中 $\{N(t), t \geqslant 0\}$ 是一强度为 $c$ 的 Poisson 过程, $0<U_{1}<U_{2}<\cdots$ 是 Poisson 过程 $N(t)$ 跳的到达时刻, $Y_{U_{1}}, Y_{U_{2}}, \cdots$ 是一列独立同分布的随机变量, 都具有参 数为 $\alpha$ 的指数分布, 即 $\Gamma(1, \alpha)$ 分布 (参见文献 $[2,10]$ ).

根据过程 $z(\lambda t)$ 和 $\sigma^{2}(t)$ 的时齐性, 过程 $\sigma^{2}(t)$ 和 $z(\lambda t)$ 的一条模拟轨道可参 见图 1.
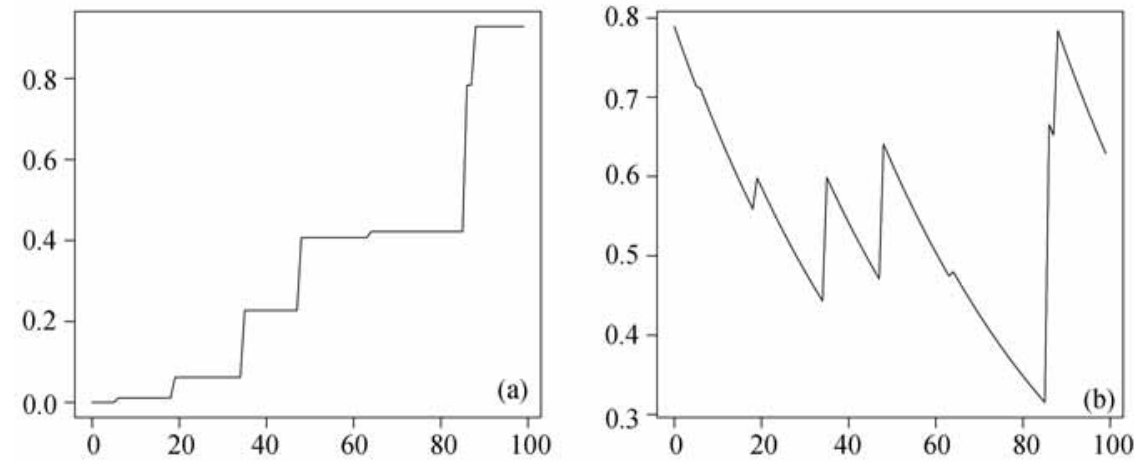

图 1 边际分布为 $\Gamma(c, \alpha)$ 分布的 $\mathrm{OU}$ 过程 $(c=3, \alpha=6, \lambda=0.02, \Delta=1)$

(a) $z(\lambda n \Delta)$ 关于 $n$ 的变化图; (b) $\sigma^{2}(n \Delta)$ 关于 $n$ 的变化图

\subsection{Gamma-OU 过程的转移密度}

讨论由方程 (1.1) 决定的过程的一些性质. 设 $(z(t), t \leqslant 0)$ 定义在概率空间 $(\Omega, \mathbb{F}, \mathbb{P})$ 上, 在文献 [11] 中证明的那样, 存在 $z$ 的一个右连左极的修正, 以下讨 论将仅考虑这一修正过程. 容易证明 Ornstein-Uhlenbeck 型方程 (1.1) 存在如下的 一个强解:

$$
\sigma^{2}(t)=e^{-\lambda t} \sigma^{2}(0)+\int_{(0, t]} e^{-\lambda(t-s)} \mathrm{d} z(\lambda s) A \#,
$$

这一解在几乎处处意义下是唯一的 ${ }^{[12]}$. 而且, 因为 $\sigma^{2}(t)$ 表示成右连左极的半鞅 的随机积分, 所以 $\mathrm{OU}$ 型过程 $\left(\sigma^{2}(t), t \geqslant 0\right)$ 本身也可设为右连左极的. 因为在任 意区间 $(0, t],(t \in(0, \infty))$ 上, $z$ 的轨道 $\mathbb{P}$ 几乎处处有限变差 ${ }^{[12]}$, 所以 (2.2) 式中 的随机积分可解释为顺向 Lebesgue-Stieltjes 积分.

引理 2.1 OU 型方程 (1.1) 存在时齐的转移函数 $P_{t}(x, B)$, 满足

$$
\int_{-\infty}^{\infty} e^{i z y} P_{t}(x, \mathrm{~d} y)=\mathbb{E}\left[\exp \left\{i z\left(e^{-\lambda t} x+\int_{(0, t]} e^{-\lambda(t-s)} \mathrm{d} z(\lambda s)\right)\right\}\right] .
$$


证明该结论可由文献 [12] 中引理 17.1 和 (2.2) 式得到.

在 (2.2) 式中, 令

$$
\tilde{\sigma}^{2}(t)=\int_{(0, t]} e^{-\lambda(t-s)} \mathrm{d} z(\lambda s) .
$$

由引理 2.1, 要求 $\sigma^{2}(t)$ 的转移函数, 对固定的 $t>0$, 先求随机变量 $\tilde{\sigma}^{2}(t)$ 的分布是 重要的. 由文献 [12] 中引理 17.1 , 随机变量 $\widetilde{\sigma}^{2}(t)$ 具有如下特征函数:

$$
\mathbb{E}\left[e^{i z \widetilde{\sigma}^{2}(t)}\right]=\exp \left\{\lambda \int_{0}^{t} \varphi\left(e^{-\lambda(t-s)} z\right) \mathrm{d} s\right\},
$$

其中 $\varphi(z)=\log (\phi(z))=\int_{0}^{\infty}\left(e^{i z x}-1\right) c \alpha e^{-\alpha x} \mathrm{~d} x, \phi(z)$ 由 (2.1) 式定义.

引理 2.2 对于固定的 $t>0$, 随机变量 $\widetilde{\sigma}^{2}(t)$ 服从复合 Poisson 分布. 具体的 讲, $\tilde{\sigma}^{2}(t)$ 可表示为如下形式:

$$
\widetilde{\sigma}^{2}(t)=\sum_{i=1}^{\tilde{N}} W_{i},
$$

其中 r.v. $\tilde{N}$ 服从强度为 $\lambda c t$ 的 Poisson 分布, $W_{1}, W_{2}, \cdots$ 是一列独立同分布的随 机变量, 它们都具有密度函数

$$
f(w)= \begin{cases}\frac{e^{-\alpha w}-e^{-\alpha w e^{\lambda t}}}{\lambda t w}, & w>0, \\ 0, & \text { 其他. }\end{cases}
$$

证明 据 (2.5) 式,

$$
\begin{aligned}
& \mathbb{E}\left[e^{i z \widetilde{\sigma}^{2}(t)}\right]=\exp \left\{\lambda \int_{0}^{t} \varphi\left(e^{-\lambda(t-s)} z\right) \mathrm{d} s\right\} \\
& =\exp \left\{\lambda \int_{0}^{t}\left(\int_{0}^{\infty}\left(e^{i e^{-\lambda(t-s)} z x}-1\right) c \alpha e^{-\alpha x} \mathrm{~d} x\right) \mathrm{d} s\right\} \\
& =\exp \left\{\lambda \int_{0}^{t}\left(\int_{0}^{\infty}\left(e^{i e^{-\lambda u} z x}-1\right) c \alpha e^{-\alpha x} \mathrm{~d} x\right) \mathrm{d} u\right\} \quad(\text { 令 } \quad u=t-s) \\
& =\exp \left\{\lambda \int_{0}^{\infty} c \alpha e^{-\alpha x}\left(\int_{0}^{t}\left(e^{i e^{-\lambda u} z x}-1\right) \mathrm{d} u\right) \mathrm{d} x\right\} \\
& =\exp \left\{\int_{0}^{\infty} c \alpha e^{-\alpha x}\left(\int_{e^{-\lambda t} x}^{x}\left(e^{i z w}-1\right) \frac{1}{w} \mathrm{~d} w\right) \mathrm{d} x\right\} \quad\left(\text { 令 } \quad w=e^{-\lambda u} x\right) \\
& =\exp \left\{\int_{0}^{\infty}\left(e^{i z w}-1\right) \frac{\int_{w}^{e^{\lambda t} w} c \alpha e^{-\alpha x} \mathrm{~d} x}{w} \mathrm{~d} w\right\} \\
& =\exp \left\{\lambda c t \int_{0}^{\infty}\left(e^{i z w}-1\right) \frac{e^{-\alpha w}-e^{-\alpha w e^{\lambda t}}}{\lambda t w} \mathrm{~d} w\right\},
\end{aligned}
$$

这是 (2.6) 式描述的复合 Poisson 分布随机变量的特征函数, 因为当 $w>0$ 时, 


$$
\begin{aligned}
\frac{e^{-\alpha w}-e^{-\alpha w e^{\lambda t}}}{\lambda t w} \geqslant 0, \text { 且 } & \\
\int_{0}^{\infty} \frac{e^{-\alpha w}-e^{-\alpha w e^{\lambda t}}}{\lambda t w} \mathrm{~d} w & =\int_{0}^{\infty} \frac{1}{\lambda t w}\left(\int_{w}^{e^{\lambda t} w} \alpha e^{-\alpha x} \mathrm{~d} x\right) \mathrm{d} w \\
& =\frac{1}{\lambda t} \int_{0}^{\infty} \alpha e^{-\alpha x}\left(\int_{e^{-\lambda t} x}^{x} \frac{1}{w} \mathrm{~d} w\right) \mathrm{d} x=1 .
\end{aligned}
$$

因此由 (2.7) 式定义的 $f(x)$ 是一密度函数, 引理得证.

引理 2.3 对于固定的 $t>0$, 随机变量 $\widetilde{\sigma}^{2}(t)$ 的分布函数为

$$
F_{\widetilde{\sigma}^{2}(t)}(x)= \begin{cases}0, & x<0, \\ e^{-\lambda c t}, & x=0, \\ e^{-\lambda c t}+\sum_{n=1}^{\infty} \frac{(\lambda c t)^{n} e^{-\lambda c t}}{n !} \int_{0}^{x} f_{n}(u) \mathrm{d} u, & x>0,\end{cases}
$$

其中 $f_{n}(x)(n \geqslant 1)$ 如下定义:

$$
f_{1}(x)=f(x), \quad f_{n}(x)=\int_{0}^{x} f(y) f_{n-1}(x-y) \mathrm{d} y .
$$

即 $f_{n}(x)$ 是 $f(x)$ 和 $f_{n-1}(x)$ 的卷积, 其中 $f(x)$ 如 (2.7) 式所定义.

证明 当 $x \leqslant 0$ 时, 由 (2.6) 式可知引理 2.3 是正确的; 当 $x>0$ 时, $\tilde{\sigma}^{2}(t)$ 的分布函数为

$$
\begin{aligned}
F_{\widetilde{\sigma}^{2}(t)}(x) & =\sum_{n=0}^{\infty} F_{\widetilde{\sigma}^{2}(t)}(x \mid \widetilde{N}=n) \mathbb{P}(\widetilde{N}=n) \\
& =e^{-\lambda c t}+\sum_{n=1}^{\infty} \frac{(\lambda c t)^{n} e^{-\lambda c t}}{n !} \mathbb{P}\left(W_{1}+W_{2}+\cdots+W_{n} \leqslant x\right) \\
& =e^{-\lambda c t}+\sum_{n=1}^{\infty} \frac{(\lambda c t)^{n} e^{-\lambda c t}}{n !} \int_{0}^{x} f_{n}(u) \mathrm{d} u,
\end{aligned}
$$

其中 $W_{i}$ 如 (2.6) 式所定义. (2.8) 式得证.

系 2.1 对于固定的 $t>0$, 随机变量 $\tilde{\sigma}^{2}(t)$ 的密度函数可表示为

$$
f_{\widetilde{\sigma}^{2}(t)}(x)=e^{-\lambda c t} \delta(x) \mathbb{I}_{\{x=0\}}+\sum_{n=1}^{\infty} \frac{(\lambda c t)^{n} e^{-\lambda c t}}{n !} f_{n}(x) \mathbb{I}_{\{x>0\}},
$$

其中 $\delta(x)$ 表示退化为 0 点的分布的密度函数 ( $\delta$ 函数详情可参见文献 $[12,13])$, $f_{n}(x)(n \geqslant 1)$ 由 (2.9) 式定义, 且 $f_{\widetilde{\sigma}^{2}(t)}(x)$ 关于 $x>0$ 是连续的.

证明 第 1 步由 (2.7) 式定义的 $f(x)$ 关于 $x>0$ 是单调递减的, 因为 $g^{\prime}(x)=$ $e^{-x}-(1+x) e^{-x}=-x e^{-x}<0$, 所以 $g(x)=(1+x) e^{-x}$ 在 $x>0$ 上单调递减, 故

$$
f^{\prime}(x)=\frac{\left(1+\alpha x e^{\lambda t}\right) e^{-\alpha x e^{\lambda t}}-(1+\alpha x) e^{-\alpha x}}{\lambda t x^{2}}<0 .
$$

第 2 步. $\forall n \in \mathbb{N}$ 和 $x>0$,

$$
f_{n}(x) \leqslant \frac{e^{\lambda t}-1}{\lambda t} \alpha .
$$


$\forall x>0$, 由 $f(x)$ 的单调性及 L'hospital 法则,

$$
f(x) \leqslant \lim _{x \rightarrow 0+} f(x)=\lim _{x \rightarrow 0+} \frac{-\alpha e^{-\alpha x}+\alpha e^{\lambda t} e^{-\alpha x e^{\lambda t}}}{\lambda t}=\frac{e^{\lambda t}-1}{\lambda t} \alpha,
$$

故

$$
f_{n}(x)=\int_{0}^{x} f(y) f_{n-1}(x-y) \mathrm{d} y \leqslant \frac{e^{\lambda t}-1}{\lambda t} \alpha \int_{0}^{\infty} f_{n-1}(y) \mathrm{d} y \leqslant \frac{e^{\lambda t}-1}{\lambda t} \alpha .
$$

第 3 步. 因为

$$
\sum_{n=1}^{\infty}\left|\frac{(\lambda c t)^{n} e^{-\lambda c t}}{n !} f_{n}(x)\right| \leqslant \sum_{n=1}^{\infty}\left(\frac{(\lambda c t)^{n} e^{-\lambda c t}}{n !} \frac{e^{\lambda t}-1}{\lambda t} \alpha\right) \leqslant \frac{e^{\lambda t}-1}{\lambda t} \alpha,
$$

所以 $\sum_{n=1}^{\infty} \frac{(\lambda c t)^{n} e^{-\lambda c t}}{n !} f_{n}(x)$ 关于 $x>0$ 是绝对一致收敛的. 由引理 2.3, $\widetilde{\sigma}^{2}(t)$ 的 密度函数为, 当 $x>0$ 时, $f_{\widetilde{\sigma}^{2}(t)}(x)=\sum_{n=1}^{\infty} \frac{(\lambda c t)^{n} e^{-\lambda c t}}{n !} f_{n}(x)$, 它是连续的. 因为 $\tilde{\sigma}^{2}(t)$ 的分布在条件 $(\tilde{N}=0)$ 时退化为一点 $x=0$, 所以 $f_{\widetilde{\sigma}^{2}(t)}(0)=e^{-\lambda c t} \delta(x)$.

定理 2.1 (2.2) 式中的 Gamma-OU 过程 $\sigma^{2}(t)$ 的时齐转移函数为

$$
P(t, y ; x, \lambda, c, \alpha)= \begin{cases}0, & y<e^{-\lambda t} x, \\ e^{-\lambda c t}, & y=e^{-\lambda t} x, \\ e^{-\lambda c t}+\sum_{n=1}^{\infty} \frac{(\lambda c t)^{n} e^{-\lambda c t}}{n !} \int_{0}^{y-e^{-\lambda t} x} f_{n}(u) \mathrm{d} u, & y>e^{-\lambda t} x,\end{cases}
$$

其中 $P(t, y ; x, \lambda, c, \alpha)$ 是 $\sigma^{2}(\cdot)$ 从 $x$ 出发, 在经过时间间隔 $t$ 之后到达 $\sigma^{2}(\cdot) \leqslant y$ 的 转移函数.

证明 等式 (2.12) 可由引理 2.3 及一些变换得到.

系 2.2 (2.2) 式中的 Gamma-OU 过程 $\sigma^{2}(t)$ 的转移密度函数可表示为

$$
\begin{aligned}
& p(t, y ; x, \lambda, c, \alpha) \\
= & e^{-\lambda c t}\left[\delta\left(y-e^{-\lambda t} x\right) \mathbb{I}_{\left\{y=e^{-\lambda t} x\right\}}+\sum_{n=1}^{\infty} \frac{(\lambda c t)^{n}}{n !} f_{n}\left(y-e^{-\lambda t} x\right) \mathbb{I}_{\left\{y>e^{-\lambda t} x\right\}}\right],
\end{aligned}
$$

其中 $p(t, y ; x, \lambda, c, \alpha)$ 为 $\sigma^{2}(\cdot)$ 从 $x$ 出发, 在经过时间间隔 $t$ 之后到达 $y$ 的转移密 度, 且对于固定的 $t, x, \lambda, c, \alpha>0, p(t, y ; x, \lambda, c, \alpha)$ 关于 $y>e^{-\lambda t} x$ 连续.

\section{3 转移密度的近似}

对于固定的 $t, y, x, \lambda, c$ 和 $\alpha$, 为了得到转移密度 $p(t, y ; x, \lambda, c, \alpha)$ 的数值, 计算 函数

$$
p(x) \triangleq \sum_{n=1}^{\infty} \frac{(\lambda c t)^{n}}{n !} f_{n}(x)
$$

的值是重要的. 我们通过计算 $p(x)$ Laplace 变换的数值逆来实现它的计算.

对于固定的 $t>0$, r.v. $\tilde{\sigma}^{2}(t)$ 服从复合 Poisson 分布, 其密度 $f_{\widetilde{\sigma}^{2}(t)}(x)=$ 
$e^{-\lambda c t}\left[\delta(x) \mathbb{I}_{\{x=0\}}+p(x) \mathbb{I}_{\{x>0\}}\right]$ 的 Laplace 变换为

$$
\begin{aligned}
\hat{f}_{\widetilde{\sigma}^{2}(t)}(s) & =\exp \left\{\lambda c t \int_{0}^{\infty}\left(e^{-s w}-1\right) \frac{e^{-\alpha w}-e^{-\alpha w e^{\lambda t}}}{\lambda t w} \mathrm{~d} w\right\} \\
& =\left(\frac{\alpha}{s+\alpha}\right)^{c}\left(\frac{\alpha e^{\lambda t}}{s+\alpha e^{\lambda t}}\right)^{-c}=e^{-\lambda c t}\left(\frac{s+\alpha e^{\lambda t}}{s+\alpha}\right)^{c},
\end{aligned}
$$

为证明第 2 个等式, 考虑函数 $\vartheta(s)=\int_{0}^{\infty} \frac{e^{-(s+\alpha) w}-e^{-\alpha w}}{w} \mathrm{~d} w$, 注意到 $\vartheta(0)=0$ 及 $\vartheta^{\prime}(s)=-(s+\alpha)^{-1}$.

由 (2.15) 式, (2.14) 式中函数 $p(x)$ 的 Laplace 变换为

$$
\hat{p}(s)=\left(\frac{s+\alpha e^{\lambda t}}{s+\alpha}\right)^{c}-1 .
$$

作为实施数值逆的基本原理, Gaver ${ }^{[14]}$ 建立了包含有限变差的 Post-Widder 公式 (参见文献 $[15,16]$ ) 的离散化变体. 令 $\Delta \hat{p}(J \theta)=\hat{p}((J+1) \theta)-\hat{p}(J \theta)$ 及 $\Delta^{k}=$ $\Delta\left(\Delta^{k-1}\right)$, 则

$$
(-1)^{J} \Delta^{J} \hat{p}(J \theta)=\sum_{k=0}^{J}(-1)^{k}\left(\begin{array}{l}
J \\
k
\end{array}\right) \hat{p}((J+k) \theta) .
$$

由文献 [14] 中 Gaver 的结论, $p(x)$ 的一个近似序列为

$$
\tilde{p}_{J}(x)=(-1)^{J} \frac{\ln 2}{x} \frac{(2 J) !}{J !(J-1) !} \Delta^{J} \hat{p}(J \ln 2 / x) .
$$

后来, Stehfest ${ }^{[17]}$ 找到了上述近似在某种意义下的最优线性组合, 也就是用 $\sum_{j=1}^{J} w(k, J) \tilde{p}_{J}(x)$ 来代替 (2.18) 式中的 $\tilde{p}_{J}(x)$ 作为 $p(x)$ 的近似以加速收玫. 以下 是文献 [17] 中 Stehfest 的结论, 也可参见文献 [15].

命题 2.1 设 $\tilde{f}_{J}(x)$ 由 (2.18) 式给出, 令

$$
p_{J}^{*}(x)=\sum_{j=1}^{J} w(k, J) \tilde{p}_{J}(x),
$$

其中

$$
w(k, J)=(-1)^{J-k} \frac{k^{J}}{k !(J-k) !},
$$

则对于所有的 $k$, 当 $J \rightarrow \infty$ 时, $p_{J}^{*}(x)-p(x)=(-1)^{J+1} \frac{\ln 2}{x} \frac{1}{J !}+o\left(\frac{1}{J !}\right)=o\left(J^{-k}\right)$.

自然地, $p(t, y ; x, \lambda, c, \alpha)$ 的一个近似序列可如下构造:

$$
\begin{aligned}
& p^{(J)}(t, y ; x, \lambda, c, \alpha) \\
= & e^{-\lambda c t}\left[\delta\left(y-e^{-\lambda t} x\right) \mathbb{I}_{\left\{y=e^{-\lambda t} x\right\}}+p_{J}^{*}\left(y-e^{-\lambda t} x\right) \mathbb{I}_{\left\{y>e^{-\lambda t} x\right\}}\right],
\end{aligned}
$$

其中 $p_{J}^{*}$ 由 (2.18)-(2.20) 式定义, $\hat{p}(x)$ 由 (2.16) 式定义.

定理 2.2 对于任意固定的 $t, \lambda, c, \alpha>0$ 和 $x>0$, 当 $J \rightarrow \infty$ 时,

$$
p^{(J)}(t, y ; x, \lambda, c, \alpha) \rightarrow p(t, y ; x, \lambda, c, \alpha),
$$

且, 收敛性对于 $(c, \alpha) \in(0, \infty) \times(0, \infty)$ 是一致的.

证明定理 2.2 可由系 2.2 和命题 2.1 直接得到. 
为方便计算, 我们采用循环算法 (参见文献 $[14,15])$ 来计算 $\tilde{p}_{J}(x)$. 当 $y>$ $e^{-\lambda t} x$ 时, 由命题 2.1 可知, 近似序列 $p^{(J)}(t, y ; x, \lambda, c, \alpha)$ 收玫到 $p(t, y ; x, \lambda, c, \alpha)$ 的 速度是较快的. 作为例子, 我们可以从图 2 看到这一现象, 这里我们固定 $t=1$ 和 $x=1$, 当 $y>e^{-\lambda t} x$ 时, 对不同的 $\lambda, c$ 和 $\alpha$, 在 $J=5$ 和 $J=8$ 之间, $p^{(J)}(t, y ; x, \lambda, c, \alpha)$ 的差别是微小的.
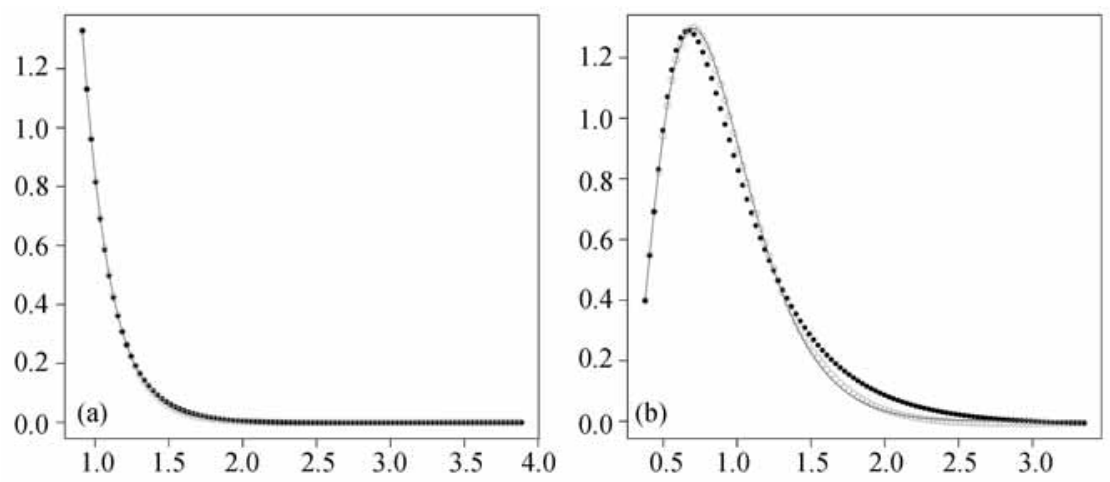

图 2 对于不同的 $\lambda$ 和边际分布 $\Gamma(c, \alpha)$, Gamma-OU 过程转移密度的近似. 在各图中,

“・・" 为 $J=3$ 时的近似, “○ $\circ \circ$ ”为 $J=5$ 时的近似, “一”为 $J=8$ 时的近似. (a) $p^{(J)}(1, y ; 1,0.1,3,6) \quad\left(y>e^{-1}\right)$; (b) $p^{(J)}(1, y ; 1,1,5,6) \quad\left(y>e^{-1}\right)$

\section{3 强度参数 $\lambda$ 的估计}

假设在时刻 $\left\{t_{k}=k \Delta, k=0,1, \cdots, n\right\}$ 观察过程, 其中 $\Delta>0$ 是固定的. 为简 单起见, 令 $X_{k}=\sigma^{2}(k \Delta)(k=0,1, \cdots, n)$. 设 $\left(x_{0}, x_{1}, \cdots, x_{n}\right)$ 为 $\left(X_{0}, X_{1}, \cdots, X_{n}\right)$ 的样本观测值. 由 Bayes 公式及离散数据可以继承的 (2.13) 式定义的转移密度的 时齐性, 似然函数将具有如下形式:

$$
l_{n}(\lambda, c, \alpha)=\left(\frac{\alpha^{c}}{\Gamma(c)} X_{0}^{c-1} e^{-\alpha X_{0}}\right)\left(\prod_{k=1}^{n} p\left(\Delta, X_{k} ; X_{k-1}, \lambda, c, \alpha\right)\right) .
$$

因为 $p\left(\Delta, x_{k} ; x_{k-1}, \lambda, c, \alpha\right)$ 的形式由 (2.13) 所定义, 所以似然函数的具体形 式由 $x_{k}-e^{-\lambda \Delta} x_{k-1}$ 的符号决定, 即由未知参数 $\lambda$ 的值所决定. 因此, 在不知道 参数 $\lambda$ 值的前提下去找 $(\lambda, c, \alpha)$ 的最大似然估计量是不可能的.

当我们设法用最大似然方法进一步估计其他参数时, 首先给出参数 $\lambda$ 的估 计是必要的, 也是重要的.

\section{1 估计 $\lambda$ 的方法}

以下定理给出了参数 $\lambda$ 的一个估计量, 这一估计量具有比弱相合性还要强 的性质.

定理 3.1 以下统计量为参数 $\lambda$ 的估计:

$$
\widehat{\lambda}_{n}=\frac{1}{\Delta} \max _{1 \leqslant k \leqslant n}\left\{\ln X_{k-1}-\ln X_{k}\right\} .
$$


而且, $\forall n \geqslant 1, \widehat{\lambda}_{n} \leqslant \lambda$ 且 $\lim _{n \rightarrow \infty} \mathbb{P}\left(\widehat{\lambda}_{n}=\lambda\right)=1$.

证明 据 (2.2)、(2.4)、(2.6) 式及 Gamma-OU 过程的时齐性, $\forall k,(k=$ $1,2, \cdots, n)$,

$$
X_{k}=e^{-\lambda \Delta} X_{k-1}+\sum_{i=1}^{\tilde{N}} W_{i},
$$

其中 r.v. $\widetilde{N}$ 服从强度为 $\lambda c \Delta$ 的 Poisson 分布, 且 $W_{1}, W_{2}, \cdots$ 是一独立同分布的正 随机变量序列, 因此

$$
\begin{gathered}
\tilde{N}=0 \Longrightarrow X_{k}=e^{-\lambda \Delta} X_{k-1} \Longleftrightarrow \lambda \Delta=\ln X_{k-1}-\ln X_{k} ; \\
\lambda \Delta>\ln X_{k-1}-\ln X_{k} \Longleftrightarrow X_{k}>e^{-\lambda \Delta} X_{k-1} \Longrightarrow \tilde{N} \geqslant 1 .
\end{gathered}
$$

首先，

$$
\widehat{\lambda}_{n}=\frac{1}{\Delta} \max _{1 \leqslant k \leqslant n}\left\{\ln X_{k-1}-\ln X_{k}\right\} \leqslant \frac{1}{\Delta} \max _{1 \leqslant k \leqslant n}(\lambda \Delta)=\lambda .
$$

要证定理 3.1, 只需证明 $\lim _{n \rightarrow \infty} \mathbb{P}\left(\widehat{\lambda}_{n}<\lambda\right)=0$. 因为

$$
\begin{aligned}
\mathbb{P}\left(\widehat{\lambda}_{n}<\lambda\right) & =\mathbb{P}\left(\max _{1 \leqslant k \leqslant n}\left\{\ln X_{k-1}-\ln X_{k}\right\}<\lambda \Delta\right) \\
& =\prod_{k=1}^{n} \mathbb{P}\left(\ln X_{k-1}-\ln X_{k}<\lambda \Delta\right) \leqslant(\mathbb{P}(\tilde{N} \geqslant 1))^{n}=\left(1-e^{-\lambda c \Delta}\right)^{n},
\end{aligned}
$$

所以 $\lim _{n \rightarrow \infty} \mathbb{P}\left(\widehat{\lambda}_{n}<\lambda\right)=0$.

\section{2 模拟实验}

由定理 3.1 的证明可知, 参数 $\lambda$ 的估计是由第 1 个非跳决定的. 为得到参数 $\lambda$ 较准确的估计, 需要取的样本容量依赖于 $\lambda$ 和 $c$ 的真值. 我们可以通过图 3 和 图 4 分别看到 $\lambda$ 和 $c$ 对 Gamma-OU 过程跳的频率的影响. 在固定的时间间隔内, 随着 $\lambda$ 或 $c$ 真值的变大, Gamma-OU 过程跳的频率也变大. 当参数 $\lambda$ 或 $c$ 的真值 较大时, 为了得到较真的 $\lambda$ 的估计值, 样本容量必须取得大一些. 然而, 在一般
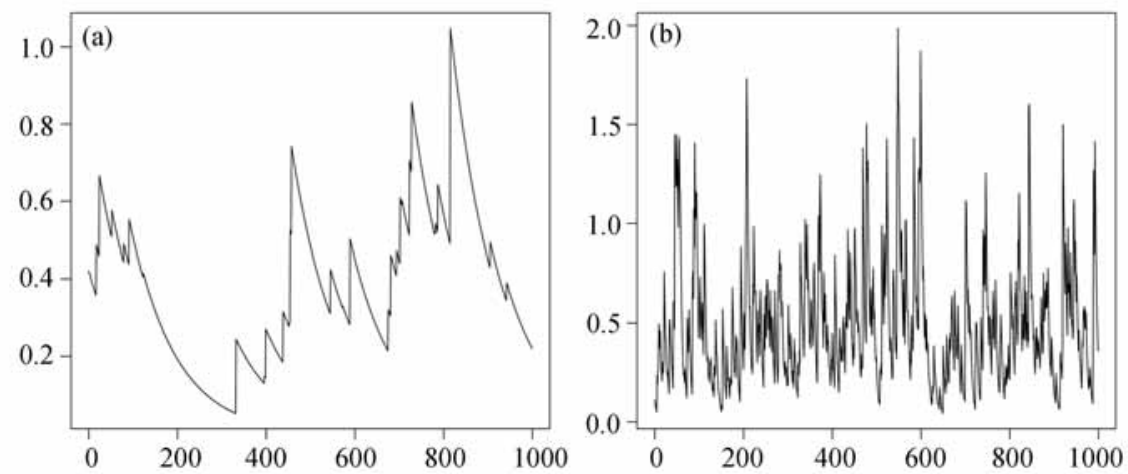

图 $3 \lambda$ 对 Gamma-OU 过程跳频率的影响 $(c=3, \alpha=6, \Delta=1)$

(a) $\sigma^{2}(n \Delta)$ 关于 $n$ 的变化图 $(\lambda=0.01)$; (b) $\sigma^{2}(n \Delta)$ 关于 $n$ 的变化图 $(\lambda=0.2)$ 
情形下，非常大的样本容量也是不必要的. 当参数 $\lambda$ 或 $c$ 的真值不是特别大时, 我们总可得到参数 $\lambda$ 的非常准确的估计. 详情可见表 1 和表 22 , 其中给出了不同 样本容量在 $n=100,200$ 和 500 下，100 次模拟所得估计的均值 (Mean) 和均方误 差的平方根 (RMSE). 每次模拟中的固定观测时间间隔为 $\Delta=1$.
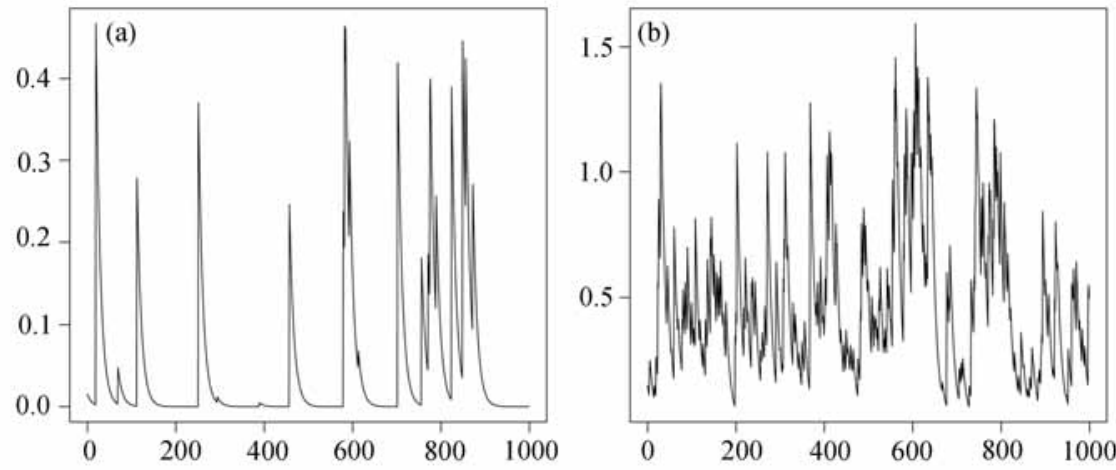

图 $4 c$ 对 Gamma-OU 过程跳频率的影响 $(\lambda=0.1, \alpha=6, \Delta=1)$

(a) $\sigma^{2}(n \Delta)$ 关于 $n$ 的变化图 $(c=0.3)$; (b) $\sigma^{2}(n \Delta)$ 关于 $n$ 的变化图 $(c=3)$

表 1 真值 $\lambda$ 对其估计的影响

\begin{tabular}{|c|c|c|c|c|c|c|c|c|}
\hline \multicolumn{3}{|c|}{ 真值 } & \multicolumn{2}{|c|}{$n=100$} & \multicolumn{2}{|c|}{$n=200$} & \multicolumn{2}{|c|}{$n=500$} \\
\hline$\lambda$ & $c$ & $\alpha$ & Mean & RMSE & Mean & RMSE & Mean & RMSE \\
\hline 0.1 & 3 & 6 & 0.1000 & 0.0000 & 0.1000 & 0.0000 & 0.1000 & 0.0000 \\
\hline 1 & 3 & 6 & 0.9997 & 0.0029 & 1.0000 & 0.0000 & 1.0000 & 0.0000 \\
\hline 1.8 & 3 & 6 & 1.7111 & 0.1400 & 1.7600 & 0.0818 & 1.7967 & 0.0133 \\
\hline 2 & 3 & 6 & 1.7907 & 0.2782 & 1.9118 & 0.1334 & 1.9855 & 0.0383 \\
\hline 2.4 & 3 & 6 & 1.9921 & 0.4928 & 2.1177 & 0.3553 & 2.3036 & 0.1518 \\
\hline
\end{tabular}

表 2 真值 $c$ 对 $\lambda$ 估计的影响

\begin{tabular}{|c|c|c|c|c|c|c|c|c|}
\hline \multicolumn{3}{|c|}{ 真值 } & \multicolumn{2}{|c|}{$n=100$} & \multicolumn{2}{|c|}{$n=200$} & \multicolumn{2}{|c|}{$n=500$} \\
\hline$\lambda$ & $c$ & $\alpha$ & Mean & RMSE & Mean & RMSE & Mean & RMSE \\
\hline 1 & 1 & 6 & 1.0000 & 0.0000 & 1.0000 & 0.0000 & 1.0000 & 0.0000 \\
\hline 1 & 3 & 6 & 0.9998 & 0.0015 & 1.0000 & 0.0000 & 1.0000 & 0.0000 \\
\hline 1 & 4 & 6 & 0.9969 & 0.0130 & 0.9991 & 0.0051 & 0.9999 & 0.0007 \\
\hline 1 & 6 & 6 & 0.9341 & 0.0918 & 0.9621 & 0.0616 & 0.9897 & 0.0229 \\
\hline 1 & 8 & 6 & 0.8424 & 0.1794 & 0.8834 & 0.1355 & 0.9471 & 0.0686 \\
\hline
\end{tabular}

据 (3.3) 式, Poisson 分布 $\tilde{N}$ 的强度为 $\lambda c \Delta$, 随着 $\lambda$ 或 $c$ 真值的递增, 跳的频 率也递增. 这就是为什么对于相同的样本容量, 随着 $\lambda$ 或 $c$ 真值的递增, 参数 $\lambda$ 估计的精确性递减. 同样的原因，高频的抽样对得到 $\lambda$ 较准确的估计是有利 
的. 由 (3.3) 式知, $\alpha$ 的值对于 Gamma-OU 过程跳的频率无影响 (直观印象可参照 图 5), 故 $\alpha$ 的真值对于得到较准确的参数 $\lambda$ 估计的样本容量是没有影响的. 现 在, 我们通过表 3 可以看到 $\alpha$ 真值对得到较精确 $\lambda$ 估计值样本容量的大小无影 响. 这里仍然是不同样本容量 $n=100,200$ 和 500 下, 100 次模拟得到的 Mean 和 RMSE. 每次模拟中, 我们固定观测的时间间隔为 $\Delta=1$.
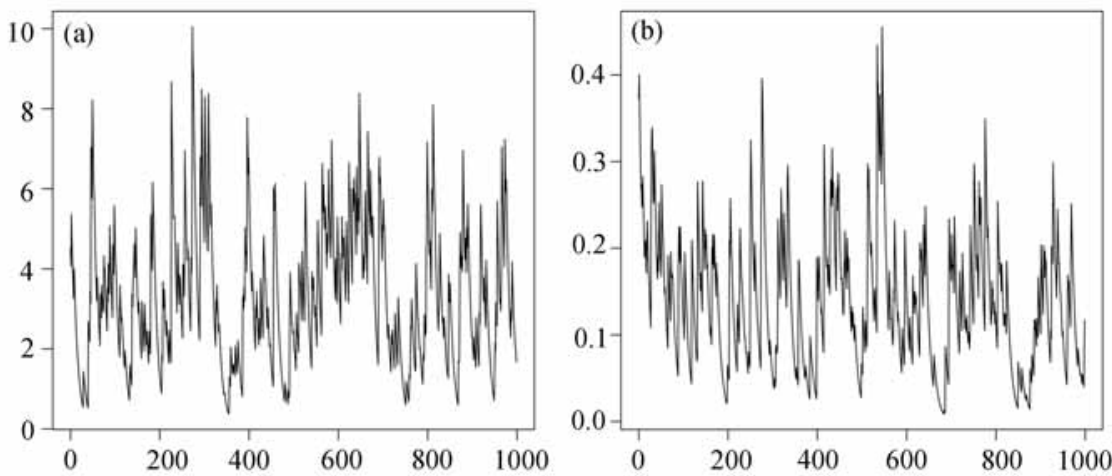

图 $5 \alpha$ 对 Gamma-OU 过程跳频率的无影响 $(\lambda=0.1, c=3, \Delta=1)$

(a) $\sigma^{2}(n \Delta)$ 关于 $n$ 的变化图 $\left(\alpha=1\right.$; (b) $\sigma^{2}(n \Delta)$ 关于 $n$ 的变化图 $(\alpha=20)$

表 3 真值 $\alpha$ 对 $\lambda$ 估计的无影响

\begin{tabular}{|c|c|c|c|c|c|c|c|c|}
\hline \multicolumn{3}{|c|}{ 真值 } & \multicolumn{2}{|c|}{$n=100$} & \multicolumn{2}{|c|}{$n=200$} & \multicolumn{2}{|c|}{$n=500$} \\
\hline$\lambda$ & $c$ & $\alpha$ & Mean & RMSE & Mean & RMSE & Mean & RMSE \\
\hline 2 & 3 & 1 & 1.7965 & 0.2815 & 1.8916 & 0.1702 & 1.9840 & 0.0399 \\
\hline 2 & 3 & 10 & 1.8250 & 0.2323 & 1.9004 & 0.1487 & 1.9839 & 0.0411 \\
\hline 2 & 3 & 20 & 1.8125 & 0.2448 & 1.9047 & 0.1585 & 1.9742 & 0.0575 \\
\hline
\end{tabular}

\section{4 似然函数}

在用上述方法将参数 $\lambda$ 估计出来之后, 现在假设它有一个已知值 $\lambda_{0}$. 从现在 开始, 我们对其他未知参数 $(c, \alpha)$ 进行最大似然估计. (3.1) 式中的项 $\frac{\alpha^{c}}{\Gamma(c)} X_{0}^{c-1} e^{-\alpha X_{0}}$ 是可以忽略掉的, 因为当 $n \rightarrow \infty$ 时, 它可由条件密度项 $p\left(\Delta, X_{k} ; X_{k-1}, \lambda, c, \alpha\right)$ 的乘积决定. 样本仅包含非条件密度 $\frac{\alpha^{c}}{\Gamma(c)} X_{0}^{c-1} e^{-\alpha X_{0}}$ 的一项, 而包含条件密度的 $n$ 项, 因此包含在样本中的 $\frac{\alpha^{c}}{\Gamma(c)} X_{0}^{c-1} e^{-\alpha X_{0}}$ 的信息不会随 $n$ 的增大而增加. 以下 所有的分布性质都是渐近性质, 所以将 $\frac{\alpha^{c}}{\Gamma(c)} X_{0}^{c-1} e^{-\alpha X_{0}}$ 项加入到似然函数中是 无意义的. 然而, (3.1) 式右端包含一个难以处理的 $\delta$ 函数. 为了去掉 $\delta(x)$, 我们 在 $\delta(x)$ 出现时乘以 $\mathrm{d} x$, 这样做对得到似然函数的最大值点不会产生影响, 且因 为 $\delta(x) \mathrm{d} x=1$ 而使似然函数得以简化. 现在, (3.1) 式右端具有较简单的形式 $\prod_{k=1}^{n}\left[e^{-\lambda_{0} c \Delta}\left(\mathbb{I}_{\left\{X_{k}=e^{-\lambda_{0} \Delta} X_{k-1}\right\}}+\sum_{n=1}^{\infty} \frac{\left(\lambda_{0} c \Delta\right)^{n}}{n !} f_{n}\left(X_{k}-e^{-\lambda_{0} \Delta} X_{k-1}\right) \mathbb{I}_{\left\{X_{k}>e^{-\lambda_{0} \Delta} X_{k-1}\right\}}\right)\right]$, 
因此对数似然函数是

$$
\begin{aligned}
L_{n}(c, \alpha)= & -n \lambda_{0} c \Delta \\
& +\sum_{k=1}^{n}\left[\ln \left(\sum_{n=1}^{\infty} \frac{\left(\lambda_{0} c \Delta\right)^{n}}{n !} f_{n}\left(X_{k}-e^{-\lambda_{0} \Delta} X_{k-1}\right)\right) \mathbb{I}_{\left\{X_{k}>e^{-\lambda_{0} \Delta} X_{k-1}\right\}}\right] .
\end{aligned}
$$

其相应的近似是

$$
L_{n}^{(J)}(c, \alpha)=-n \lambda_{0} c \Delta+\sum_{k=1}^{n} \ln \left(p_{J}^{*}\left(X_{k}-e^{-\lambda_{0} \Delta} X_{k-1}\right)\right) \mathbb{I}_{\left\{X_{k}>e^{-\lambda_{0} \Delta} X_{k-1}\right\}} .
$$

令

$$
\begin{aligned}
& h\left(\Delta, X_{k} ; X_{k-1}, c, \alpha\right):=\ln \left(\sum_{n=1}^{\infty} \frac{\left(\lambda_{0} c \Delta\right)^{n}}{n !} f_{n}\left(X_{k}-e^{-\lambda_{0} \Delta} X_{k-1}\right)\right), \\
& g\left(\Delta, X_{k} ; X_{k-1}, c, \alpha\right):=-\lambda_{0} c \Delta+h\left(\Delta, X_{k} ; X_{k-1}, c, \alpha\right) \mathbb{I}_{\left\{X_{k}>e^{-\lambda_{0} \Delta} X_{k-1}\right\}},
\end{aligned}
$$

则 $L_{n}(c, \alpha)=\sum_{k=1}^{n} g\left(\Delta, X_{k} ; X_{k-1}, c, \alpha\right)$.

以下引理证明了 $g\left(\Delta, x_{k} ; x_{k-1}, c, \alpha\right)$ 的连续可微性.

引理 4.1 $\forall k=1,2, \cdots, n$, 对固定的时间间隔 $\Delta>0, \lambda_{0}>0, x_{k}$ 和 $x_{k-1}$, $g\left(\Delta, x_{k} ; x_{k-1}, c, \alpha\right)$ 关于 $(c, \alpha) \in(0, \infty) \times(0, \infty)$ 是 3 次连续可微的.

证明 $\forall k=1,2, \cdots, n$, 对于固定的时间间隔 $\Delta>0, \lambda_{0}>0, x_{k}$ 和 $x_{k-1}$, 仅需证明 $h\left(\Delta, x_{k} ; x_{k-1}, c, \alpha\right)$ 关于 $(c, \alpha) \in(0, \infty) \times(0, \infty) 3$ 次连续可微即可. $\forall(c, \alpha) \in(0, \infty) \times(0, \infty)$, 存在 $c_{u}>0$ 和 $\alpha_{u}>0$, 使得 $(c, \alpha) \in\left(0, c_{u}\right) \times\left(0, \alpha_{u}\right)$. 只 要 $h\left(\Delta, x_{k} ; x_{k-1}, c, \alpha\right)$ 关于 $c$ 和 $\alpha$ 的 3 次导数在 $(c, \alpha) \in\left(0, c_{u}\right) \times\left(0, \alpha_{u}\right)$ 点存在, 其连续性是显然的. 证明可分两步进行.

第 1 步. $\forall n \geqslant 1$ 和 $x>0$, 对固定的时间间隔 $\Delta>0$, 由 (2.9) 式定义的 $f_{n}(x)$ 关于 $\alpha \in\left(0, \alpha_{u}\right)$ 三阶以内的偏导数是有界的.

由 (2.11) 式, $\forall n \in \mathbb{N}$,

$$
\left|f_{n}(x)\right| \leqslant \frac{e^{\lambda_{0} \Delta}-1}{\lambda_{0} \Delta} \alpha \leqslant \frac{e^{\lambda_{0} \Delta}-1}{\lambda_{0} \Delta} \alpha_{u},
$$

利用数学归纳法, 可以证明 $\forall n \in \mathbb{N}$,

$$
\begin{aligned}
\left|\frac{\partial f_{n}(x)}{\partial \alpha}\right| \leqslant & \frac{n}{\lambda_{0} \Delta}\left(1+e^{\lambda_{0} \Delta}\right), \\
\left|\frac{\partial^{2} f_{n}(x)}{\partial \alpha^{2}}\right| \leqslant & \frac{n}{\lambda_{0} \Delta}\left(1+e^{2 \lambda_{0} \Delta}\right) x+\frac{n(n-1)}{\left(\lambda_{0} \Delta\right)^{2}}\left(1+e^{\lambda_{0} \Delta}\right)^{2} x, \\
\left|\frac{\partial^{3} f_{n}(x)}{\partial \alpha^{3}}\right| \leqslant & \frac{n}{\lambda_{0} \Delta}\left(1+e^{3 \lambda_{0} \Delta}\right) x^{2}+\frac{3 n(n-1)}{\left(\lambda_{0} \Delta\right)^{2}}\left(1+e^{\lambda_{0} \Delta}\right)\left(1+e^{2 \lambda_{0} \Delta}\right) x^{2} \\
& +\frac{n(n-1)(n-2)}{\left(\lambda_{0} \Delta\right)^{3}}\left(1+e^{\lambda_{0} \Delta}\right)^{3} x^{2} .
\end{aligned}
$$

作为例子, 仅证明 (4.5) 式. 当 $n=1$ 时,

$$
\left|\frac{\partial^{2} f_{1}(x)}{\partial \alpha^{2}}\right|=\left|\frac{\partial^{2} f(x)}{\partial \alpha^{2}}\right|=\frac{1}{\lambda_{0} \Delta}\left|-e^{2 \lambda_{0} \Delta} x e^{-\alpha x e^{\lambda_{0} \Delta}}+x e^{-\alpha x}\right| \leqslant \frac{1}{\lambda_{0} \Delta}\left(1+e^{2 \lambda_{0} \Delta}\right) x,
$$


满足 (4.5) 式. 假设 (4.4) 式得证, 且 (4.5) 式对 $n-1$ 的情形成立, 则对 $n$ 的情形 为

$$
\begin{aligned}
\left|\frac{\partial^{2} f_{n}(x)}{\partial \alpha^{2}}\right|= & \mid \int_{0}^{x} \frac{\partial^{2} f(y)}{\partial \alpha^{2}} f_{n-1}(x-y) \mathrm{d} y+2 \int_{0}^{x} \frac{\partial f(y)}{\partial \alpha} \frac{\partial f_{n-1}(x-y)}{\partial \alpha} \mathrm{d} y \\
& +\int_{0}^{x} f(y) \frac{\partial^{2} f_{n-1}(x-y)}{\partial \alpha^{2}} \mathrm{~d} y \mid \\
\leqslant & \frac{1}{\lambda_{0} \Delta}\left(1+e^{2 \lambda_{0} \Delta}\right) x+2\left(\frac{1}{\lambda_{0} \Delta}\left(1+e^{\lambda_{0} \Delta}\right)\right)\left(\frac{n-1}{\lambda_{0} \Delta}\left(1+e^{\lambda_{0} \Delta}\right)\right) x \\
& +\frac{n-1}{\lambda_{0} \Delta}\left(1+e^{2 \lambda_{0} \Delta}\right) x+\frac{(n-1)(n-2)}{\left(\lambda_{0} \Delta\right)^{2}}\left(1+e^{\lambda_{0} \Delta}\right)^{2} x \\
= & \frac{n}{\lambda_{0} \Delta}\left(1+e^{2 \lambda_{0} \Delta}\right) x+\frac{n(n-1)}{\left(\lambda_{0} \Delta\right)^{2}}\left(1+e^{\lambda_{0} \Delta}\right)^{2} x
\end{aligned}
$$

(4.5) 式得证.

第 2 步. $\forall k=1,2, \cdots, n$, 对任意固定的 $x_{k-1}, x_{k}$ 和时间间隔 $\Delta>0$, $h\left(\Delta, x_{k} ; x_{k-1}, c, \alpha\right)$ 关于 $c$ 和 $\alpha$ 三阶以内的偏导数在 $(c, \alpha) \in\left(0, c_{u}\right) \times\left(0, \alpha_{u}\right)$ 点存 在.

$$
\begin{aligned}
\text { 令 } z_{k}:=x_{k}-e^{-\lambda_{0} \Delta} x_{k-1} \text { 及 } \\
A_{0}\left(z_{k}, c, \alpha\right):=\sum_{n=1}^{\infty} \frac{\left(\lambda_{0} c \Delta\right)^{n}}{n !} f_{n}\left(z_{k}\right), \\
A_{i}\left(z_{k}, c, \alpha\right):=\sum_{n=i}^{\infty} \frac{\left(\lambda_{0} c \Delta\right)^{n-i}}{(n-i) !} f_{n}\left(z_{k}\right), \quad i=1,2,3, \\
B_{i}\left(z_{k}, c, \alpha\right):=\sum_{n=1}^{\infty} \frac{\left(\lambda_{0} c \Delta\right)^{n}}{n !} \frac{\partial^{i} f_{n}}{\partial \alpha^{i}}\left(z_{k}\right), \quad i=1,2,3, \\
C_{11}\left(z_{k}, c, \alpha\right):=\sum_{n=1}^{\infty} \frac{\left(\lambda_{0} c \Delta\right)^{n-1}}{(n-1) !} \frac{\partial f_{n}}{\partial \alpha}\left(z_{k}\right), \\
C_{i j}\left(z_{k}, c, \alpha\right):=\sum_{n=i}^{\infty} \frac{\left(\lambda_{0} c \Delta\right)^{n-i}}{(n-i) !} \frac{\partial^{j} f_{n}}{\partial \alpha^{j}}\left(z_{k}\right), \quad(i, j)=(1,2) \text { 或 }(2,1) .
\end{aligned}
$$

据 (4.3)-(4.6) 式,

$$
\begin{aligned}
& \left|\frac{\left(\lambda_{0} c \Delta\right)^{n}}{n !} f_{n}\left(z_{k}\right)\right| \leqslant D_{0} \alpha_{u} \frac{\left(\lambda_{0} c_{u} \Delta\right)^{n}}{n !}, \\
& \left|\frac{\left(\lambda_{0} c \Delta\right)^{n-i}}{(n-i) !} f_{n}\left(z_{k}\right)\right| \leqslant D_{0} \alpha_{u} \frac{\left(\lambda_{0} c_{u} \Delta\right)^{n-i}}{(n-i) !}, \quad i=1,2,3, \\
& \left|\frac{\left(\lambda_{0} c \Delta\right)^{n}}{n !} \frac{\partial f_{n}}{\partial \alpha}\left(z_{k}\right)\right| \leqslant D_{1} \frac{\left(\lambda_{0} c_{u} \Delta\right)^{n}}{(n-1) !}, \\
& \left|\frac{\left(\lambda_{0} c \Delta\right)^{n}}{n !} \frac{\partial^{2} f_{n}}{\partial \alpha^{2}}\left(z_{k}\right)\right| \leqslant D_{2} \frac{\left(\lambda_{0} c_{u} \Delta\right)^{n}}{(n-1) !} z_{k}+D_{1}^{2} \frac{\left(\lambda_{0} c_{u} \Delta\right)^{n}}{(n-2) !} z_{k},
\end{aligned}
$$




$$
\begin{gathered}
\left|\frac{\left(\lambda_{0} c \Delta\right)^{n}}{n !} \frac{\partial^{3} f_{n}}{\partial \alpha^{3}}\left(z_{k}\right)\right| \leqslant D_{3} \frac{\left(\lambda_{0} c_{u} \Delta\right)^{n}}{(n-1) !} z_{k}^{2}+D_{1} D_{2} \frac{\left(\lambda_{0} c_{u} \Delta\right)^{n}}{(n-2) !} z_{k}^{2}+D_{1}^{3} \frac{\left(\lambda_{0} c_{u} \Delta\right)^{n}}{(n-3) !} z_{k}^{2}, \\
\left|\frac{\left(\lambda_{0} c \Delta\right)^{n-1}}{(n-1) !} \frac{\partial f_{n}}{\partial \alpha}\left(z_{k}\right)\right| \leqslant D_{1} \frac{\left(\lambda_{0} c_{u} \Delta\right)^{n-1}}{(n-2) !}+D_{1} \frac{\left(\lambda_{0} c_{u} \Delta\right)^{n-1}}{(n-1) !} \\
\left|\frac{\left(\lambda_{0} c \Delta\right)^{n-1}}{(n-1) !} \frac{\partial^{2} f_{n}}{\partial \alpha^{2}}\left(z_{k}\right)\right| \leqslant D_{2} \frac{\left(\lambda_{0} c_{u} \Delta\right)^{n-1}}{(n-2) !} z_{k}+D_{2} \frac{\left(\lambda_{0} c_{u} \Delta\right)^{n-1}}{(n-1) !} z_{k} \\
\quad+D_{1}^{2} \frac{\left(\lambda_{0} c_{u} \Delta\right)^{n-1}}{(n-3) !} z_{k}+2 D_{1}^{2} \frac{\left(\lambda_{0} c_{u} \Delta\right)^{n-1}}{(n-2) !} z_{k}, \\
\left|\frac{\left(\lambda_{0} c \Delta\right)^{n-2}}{(n-2) !} \frac{\partial f_{n}}{\partial \alpha}\left(z_{k}\right)\right| \leqslant D_{1} \frac{\left(\lambda_{0} c_{u} \Delta\right)^{n-2}}{(n-3) !}+2 D_{1} \frac{\left(\lambda_{0} c_{u} \Delta\right)^{n-2}}{(n-2) !},
\end{gathered}
$$

其中 $D_{0}=\frac{e^{\lambda_{0} \Delta}-1}{\lambda_{0} \Delta}, D_{i}=\frac{1}{\lambda_{0} \Delta}\left(1+e^{\lambda_{0} \Delta}\right), i=1,2,3 . \forall k=1,2, \cdots, n$, 上述不等式右 端项的无穷级数都是收玫的, 所以 $A_{0}\left(z_{k}, c, \alpha\right), A_{i}\left(z_{k}, c, \alpha\right), B_{i}\left(z_{k}, c, \alpha\right)(i=1,2,3)$, $C_{11}\left(z_{k}, c, \alpha\right)$ 和 $C_{i j}\left(z_{k}, c, \alpha\right)((i, j)=(1,2)$ 或 $(2,1))$ 均关于 $(c, \alpha) \in\left(0, c_{u}\right) \times\left(0, \alpha_{u}\right)$ 一致收敛.

因为

$$
\begin{aligned}
& \frac{\partial h}{\partial c}=\lambda_{0} \Delta \frac{A_{1}}{A_{0}}, \quad \frac{\partial h}{\partial \alpha}=\frac{B_{1}}{A_{0}}, \\
& \frac{\partial^{2} h}{\partial c^{2}}=\left(\lambda_{0} \Delta\right)^{2}\left[\frac{A_{2}}{A_{0}}-\left(\frac{A_{1}}{A_{0}}\right)^{2}\right], \quad \frac{\partial^{2} h}{\partial c \partial \alpha}=\lambda_{0} \Delta\left[\frac{C_{11}}{A_{0}}-\frac{A_{1}}{A_{0}} \frac{B_{1}}{A_{0}}\right], \\
& \frac{\partial^{2} h}{\partial \alpha^{2}}=\frac{B_{2}}{A_{0}}-\left(\frac{B_{1}}{A_{0}}\right)^{2}, \quad \frac{\partial^{3} h}{\partial c^{3}}=\left(\lambda_{0} \Delta\right)^{3}\left[\frac{A_{3}}{A_{0}}-3 \frac{A_{1}}{A_{0}} \frac{A_{2}}{A_{0}}+2\left(\frac{A_{1}}{A_{0}}\right)^{3}\right], \\
& \frac{\partial^{3} h}{\partial c^{2} \partial \alpha}=\left(\lambda_{0} \Delta\right)^{2}\left[\frac{C_{21}}{A_{0}}-2 \frac{A_{1}}{A_{0}} \frac{C_{11}}{A_{0}}-\frac{B_{1}}{A_{0}} \frac{A_{2}}{A_{0}}+2\left(\frac{A_{1}}{A_{0}}\right)^{2} \frac{B_{1}}{A_{0}}\right] \\
& \frac{\partial^{3} h}{\partial c \partial \alpha^{2}}=\left(\lambda_{0} \Delta\right)\left[\frac{C_{12}}{A_{0}}-2 \frac{B_{1}}{A_{0}} \frac{C_{11}}{A_{0}}-\frac{A_{1}}{A_{0}} \frac{B_{2}}{A_{0}}+2 \frac{A_{1}}{A_{0}}\left(\frac{B_{1}}{A_{0}}\right)^{2}\right] \\
& \frac{\partial^{3} h}{\partial \alpha^{3}}=\frac{B_{3}}{A_{0}}-3 \frac{B_{1}}{A_{0}} \frac{B_{2}}{A_{0}}+2\left(\frac{B_{1}}{A_{0}}\right)^{3},
\end{aligned}
$$

其中 $A_{i}(i=0,1,2,3), B_{i}(i=1,2,3)$ 和 $C_{i j}((i, j)=(1,1),(1,2)$ 或 $(2,1))$ 分 别是 $A_{i}\left(z_{k}, c, \alpha\right)(i=0,1,2,3), B_{i}\left(z_{k}, c, \alpha\right)(i=1,2,3)$ 和 $C_{i j}\left(z_{k}, c, \alpha\right)((i, j)=$ $(1,1),(1,2)$ 或 $(2,1))$ 的缩写式, 所以 $\forall(c, \alpha) \in(0, \infty) \times(0, \infty)$, 存在 $c_{u}>c$ 和 $\alpha_{u}>\alpha$, 使得 (4.7)-(4.12) 式都存在. 引理得证.

\section{5 最大似然估计}

\section{1 矩条件}

令 $Z_{k}:=X_{k}-e^{-\lambda_{0} \Delta} X_{k-1}, i=1,2, \cdots, n$, 它具有同 (2.4) 式所定义的随机 变量 $\tilde{\sigma}^{2}(\Delta)$ 相同的分布. 设 $\left(c_{0}, \alpha_{0}\right)$ 为参数 $(c, \alpha)$ 的真值. 由 (4.7)-(4.12) 式及 Hölder 不等式, $\mathbb{E}\left[\left|\frac{\partial g}{\partial c}\right|\right], \mathbb{E}\left[\left|\frac{\partial g}{\partial \alpha}\right|\right], \mathbb{E}\left[\left|\frac{\partial^{2} g}{\partial c^{2}}\right|\right], \mathbb{E}\left[\left|\frac{\partial^{2} g}{\partial c \partial \alpha}\right|\right], \mathbb{E}\left[\left|\frac{\partial^{2} g}{\partial \alpha^{2}}\right|\right], \mathbb{E}\left[\left|\frac{\partial^{3} g}{\partial c^{3}}\right|\right], \mathbb{E}\left[\left|\frac{\partial^{3} g}{\partial c^{2} \partial \alpha}\right|\right]$, 
$\mathbb{E}\left[\left|\frac{\partial^{3} g}{\partial c \partial \alpha^{2}}\right|\right]$ 和 $\mathbb{E}\left[\left|\frac{\partial^{3} g}{\partial \alpha^{3}}\right|\right]$ 均在 $\left(c_{0}, \alpha_{0}\right)$ 点存在的充分必要条件是 $\mathbb{E}\left[\left|\frac{A_{i}}{A_{0}} \mathbb{I}_{\left\{Z_{1}>0\right\}}\right|^{4-i}\right]$, $\mathbb{E}\left[\left|\frac{B_{i}}{A_{0}} \mathbb{I}_{\left\{Z_{1}>0\right\}}\right|^{4-i}\right](i=1,2,3), \mathbb{E}\left[\left|\frac{C_{i j}}{A_{0}} \mathbb{I}_{\left\{Z_{1}>0\right\}}\right|^{4-(i+j)}\right]((i, j)=(1,1),(1,2)$ 或 $(2,1))$ 均在 $\left(c_{0}, \alpha_{0}\right)$ 点存在.

事实上, 对于固定的 $\Delta>0$, 由 Hölder 不等式和 (2.10) 式, 易证 $\mathbb{E}\left[\left|\frac{A_{1}}{A_{0}} \mathbb{I}_{\left\{Z_{1}>0\right\}}\right|^{3}\right]$ 和 $\mathbb{E}\left[\left|\frac{A_{2}}{A_{0}} \mathbb{I}_{\left\{Z_{1}>0\right\}}\right|^{2}\right]$ 的存在性. 例如,

$$
\begin{aligned}
\mathbb{E}\left[\left|\frac{A_{1}}{A_{0}} \mathbb{I}_{\left\{Z_{1}>0\right\}}\right|^{3}\right] & =\int_{0}^{\infty} \frac{\left(\sum_{n=1}^{\infty} \frac{\left(\lambda_{0} c_{0} \Delta\right)^{n-1}}{(n-1) !} f_{n}(x)\right)^{3}}{\left(\sum_{n=1}^{\infty} \frac{\left(\lambda_{0} c_{0} \Delta\right)^{n}}{n !} f_{n}(x)\right)^{2}} \mathrm{~d} x \\
& \leqslant \int_{0}^{\infty} \frac{\sum_{n=1}^{\infty}\left(\frac{\left(\lambda_{0} c_{0} \Delta\right)^{n-1} n}{(n-1) !} f_{n}(x)\right)^{3}\left(\sum_{n=1}^{\infty} \frac{1}{n^{\frac{3}{2}}}\right)^{2}}{\left(\sum_{n=1}^{\infty} \frac{\left(\lambda_{0} c_{0} \Delta\right)^{n}}{n !} f_{n}(x)\right)^{2}} \mathrm{~d} x \\
& \leqslant\left(\sum_{n=1}^{\infty} \frac{1}{n^{\frac{3}{2}}}\right)^{2} \int_{0}^{\infty} \sum_{n=1}^{\infty} \frac{\left(\frac{\left(\lambda_{0} c_{0} \Delta\right)^{n-1} n}{n-1) !} f_{n}(x)\right)^{3}}{\left(\frac{\left(\lambda_{0} c_{0} \Delta\right)^{n}}{n !} f_{n}(x)\right)^{2}} \mathrm{~d} x \\
& =\left(\sum_{n=1}^{\infty} \frac{1}{n^{\frac{3}{2}}}\right)^{2} \int_{0}^{\infty} \sum_{n=1}^{\infty} \frac{n^{5}\left(\lambda_{0} c_{0} \Delta\right)^{n-3}}{(n-1) !} f_{n}(x) \mathrm{d} x \\
& =\left(\sum_{n=1}^{\infty} \frac{1}{n^{\frac{3}{2}}}\right)^{2} \sum_{n=1}^{\infty} \frac{n^{5}\left(\lambda_{0} c_{0} \Delta\right)^{n-3}}{(n-1) !}<\infty .
\end{aligned}
$$

$\mathbb{E}\left[\left|\frac{A_{3}}{A_{0}} \mathbb{I}_{\left\{Z_{1}>0\right\}}\right|\right]<\infty$ 是显然的, 这是因为

$$
\mathbb{E}\left[\left|\frac{A_{3}}{A_{0}} \mathbb{I}_{\left\{Z_{1}>0\right\}}\right|\right]=\sum_{n=3}^{\infty} \frac{\left(\lambda_{0} c_{0} \Delta\right)^{n-3}}{(n-3) !} \int_{0}^{\infty} f_{n}(x) \mathrm{d} x<\infty .
$$

为了证明 $\mathbb{E}\left[\left|\frac{B_{i}}{A_{0}} \mathbb{I}_{\left\{Z_{1}>0\right\}}\right|^{4-i}\right](i=1,2,3)$ 和 $\mathbb{E}\left[\left|\frac{C_{i j}}{A_{0}} \mathbb{I}_{\left\{Z_{1}>0\right\}}\right|^{4-(i+j)}\right]((i, j)=$ $(1,1),(1,2)$ 或 $(2,1))$ 的存在性, 我们首先证明如下一些不等式:

引理 5.1 对于固定的时间间隔 $\Delta>0$ 和固定的 $\lambda_{0}, c>0, \forall n \geqslant 1$ 及 $x>0$, 由 (2.9) 式所定义的函数 $f_{n}(x)$ 关于 $\alpha$ 三阶以内的偏导数满足如下一些不等式: 当 $\alpha \geqslant 1$ 时,

$$
\begin{aligned}
& \left|\frac{\partial f_{n}(x)}{\partial \alpha}\right| \leqslant n\left(1+x e^{\lambda_{0} \Delta}\right) f_{n}(x) \\
& \left|\frac{\partial^{2} f_{n}(x)}{\partial \alpha^{2}}\right| \leqslant\left[n\left(1+x^{2} e^{2 \lambda_{0} \Delta}\right)+n(n-1)\left(1+x e^{\lambda_{0} \Delta}\right)^{2}\right] f_{n}(x)
\end{aligned}
$$

当 $\alpha \geqslant 2^{\frac{2}{3}}$ 时,

$$
\begin{aligned}
\left|\frac{\partial^{3} f_{n}(x)}{\partial \alpha^{3}}\right| \leqslant & {\left[n\left(1+x^{3} e^{3 \lambda_{0} \Delta}\right)+3 n(n-1)\left(1+x e^{\lambda_{0} \Delta}\right)\left(1+x^{2} e^{2 \lambda_{0} \Delta}\right)\right.} \\
& \left.+n(n-1)(n-2)\left(1+x e^{\lambda_{0} \Delta}\right)^{3}\right] f_{n}(x) .
\end{aligned}
$$

证明 当 $x \geqslant \frac{\lambda_{0} \Delta}{\alpha\left(e^{\lambda_{0} \Delta}-1\right)}$ 时, 因为 $e^{-\alpha x} \geqslant e^{\lambda_{0} \Delta} e^{-\alpha x e^{\lambda_{0} \Delta}}$, 所以

$$
\begin{aligned}
\left|\frac{\partial f(x)}{\partial \alpha}\right| & =\frac{1}{\lambda_{0} \Delta}\left(e^{-\alpha x}-e^{\lambda_{0} \Delta} e^{-\alpha x e^{\lambda_{0} \Delta}}\right) \\
& \leqslant x e^{\lambda_{0} \Delta} \frac{e^{-\alpha x}-e^{-\alpha x e^{\lambda_{0} \Delta}}}{\lambda_{0} \Delta x}=x e^{\lambda_{0} \Delta} f(x),
\end{aligned}
$$


其中 $f(x)$ 由 (2.7) 式定义. 对于固定的 $\alpha \geqslant 1$, 考虑函数 $g(y)=(1+y) e^{-\alpha y}$, 当 $y>0$ 时, 因为 $g^{\prime}(y)=(1-\alpha-\alpha y) e^{-\alpha y} \leqslant 0$, 所以 $g(y)$ 是单调递减的, 故

$$
\begin{aligned}
x & <x e^{\lambda_{0} \Delta} \Longrightarrow\left(1+x e^{\lambda_{0} \Delta}\right) e^{-\alpha x e^{\lambda_{0} \Delta}} \\
& \leqslant(1+x) e^{-\alpha x} \Longrightarrow x\left(e^{\lambda_{0} \Delta} e^{-\alpha x e^{\lambda_{0} \Delta}}-e^{-\alpha x}\right) \leqslant e^{-\alpha x}-e^{-\alpha x e^{\lambda_{0} \Delta}} .
\end{aligned}
$$

因此, 当 $x<\frac{\lambda_{0} \Delta}{\alpha\left(e^{\lambda^{\lambda_{0}} \Delta}-1\right)}$ 时, $e^{-\alpha x}<e^{\lambda_{0} \Delta} e^{-\alpha x e^{\lambda_{0} \Delta}}$,

$$
\left|\frac{\partial f(x)}{\partial \alpha}\right|=\frac{1}{\lambda_{0} \Delta}\left(e^{\lambda_{0} \Delta} e^{-\alpha x e^{\lambda_{0} \Delta}}-e^{-\alpha x}\right)=\frac{x\left(e^{\lambda_{0} \Delta} e^{-\alpha x e^{\lambda_{0} \Delta}}-e^{-\alpha x}\right)}{\lambda_{0} \Delta x} \leqslant f(x) .
$$

由 (5.4) 及 (5.5) 式,

$$
\left|\frac{\partial f(x)}{\partial \alpha}\right| \leqslant\left(1+x e^{\lambda_{0} \Delta}\right) f(x) .
$$

类似地, 当 $x \geqslant \frac{2 \lambda_{0} \Delta}{\alpha\left(e^{\lambda_{0} \Delta}-1\right)}$ 时, 可以证明 $\left|\frac{\partial^{2} f(x)}{\partial \alpha^{2}}\right| \leqslant x^{2} e^{2 \lambda_{0} \Delta} f(x)$; 当 $x<\frac{2 \lambda_{0} \Delta}{\alpha\left(e^{\lambda_{0} \Delta}-1\right)}$ 时, 可以通过考虑函数 $g(y)=\left(1+y^{2}\right) e^{-\alpha y}$ 当 $y>0$ 时的单调递减性来证明 $\left|\frac{\partial^{2} f(x)}{\partial \alpha^{2}}\right| \leqslant f(x)$, 因此

$$
\left|\frac{\partial^{2} f(x)}{\partial \alpha^{2}}\right| \leqslant\left(1+x^{2} e^{2 \lambda_{0} \Delta}\right) f(x) .
$$

当 $x \geqslant \frac{3 \lambda_{0} \Delta}{\alpha\left(e^{\lambda_{0} \Delta}-1\right)}$ 时, $\left|\frac{\partial^{3} f(x)}{\partial \alpha^{3}}\right| \leqslant x^{3} e^{3 \lambda_{0} \Delta} f(x)$ 可像 (5.4) 式那样证明; 对于固定的 $\alpha \geqslant 2^{\frac{2}{3}}$, 考虑函数 $g(y)=\left(1+y^{3}\right) e^{-\alpha y}$, 当 $y \geqslant 0$ 时, $g^{\prime}(y)=\left(3 y^{2}-\alpha-\alpha y^{3}\right) e^{-\alpha y}$, 令 $h(y)=3 y^{2}-\alpha-\alpha y^{3}$, 因为

$$
h^{\prime}(y)=3 y(2-\alpha y)=0 \Longrightarrow y=0 \text { 或 } y=\frac{2}{\alpha} \text {. }
$$

所以

$$
\begin{aligned}
\max _{y \geqslant 0} h(y) & =\max \left\{h(0), h\left(\frac{2}{\alpha}\right), h(+\infty)\right\} \\
& =\max \left\{-\alpha, \frac{4}{\alpha^{2}}-\alpha,-\infty\right\}=\frac{4}{\alpha^{2}}-\alpha \leqslant \frac{4}{\left(2^{\frac{2}{3}}\right)^{2}}-2^{\frac{2}{3}}=0 .
\end{aligned}
$$

因此, $g^{\prime}(y) \leqslant 0$, 且 $g(y)$ 当 $y \geqslant 0$ 时是单调递减的, 故可利用与证明 (5.5) 式相同 的方法, 得到当 $x<\frac{3 \lambda_{0} \Delta}{\alpha\left(e^{\lambda_{0} \Delta}-1\right)}$ 时, $\left|\frac{\partial^{3} f(x)}{\partial \alpha^{3}}\right| \leqslant f(x)$, 故

$$
\left|\frac{\partial^{3} f(x)}{\partial \alpha^{3}}\right| \leqslant\left(1+x^{3} e^{3 \lambda_{0} \Delta}\right) f(x) .
$$

(5.6)-(5.8) 式分别是 (5.1)-(5.3) 式当 $n=1$ 时的特殊情形. 用同证明 (4.4)-(4.6) 式 类似的数学归纳法易证得 (5.1)-(5.3) 式.

引理 5.2 对于固定的时间间隔 $\Delta>0$ 和固定的 $\lambda_{0}, c, \alpha>0, \forall n \in \mathbb{N}$,

$$
\begin{aligned}
& \mathbb{E}\left[V_{n}\right]=n \frac{1-e^{-\lambda_{0} \Delta}}{\lambda_{0} \Delta \alpha}, \\
& \mathbb{E}\left[V_{n}^{2}\right]=n(n-1) \frac{\left(1-e^{-\lambda_{0} \Delta}\right)^{2}}{\left(\lambda_{0} \Delta \alpha\right)^{2}}+n \frac{1-e^{-2 \lambda_{0} \Delta}}{\lambda_{0} \Delta \alpha^{2}}, \\
& \mathbb{E}\left[V_{n}^{3}\right]=n(n-1)(n-2) \frac{\left(1-e^{-\lambda_{0} \Delta}\right)^{3}}{\left(\lambda_{0} \Delta \alpha\right)^{3}}+n \frac{2\left(1-e^{-3 \lambda_{0} \Delta}\right)}{\lambda_{0} \Delta \alpha^{3}}
\end{aligned}
$$




$$
+3 n(n-1) \frac{\left(1-e^{-\lambda_{0} \Delta}\right)^{2}\left(1+e^{-\lambda_{0} \Delta}\right)}{\left(\lambda_{0} \Delta\right)^{2} \alpha^{3}},
$$

$$
\begin{aligned}
\mathbb{E}\left[V_{n}^{4}\right]= & n(n-1)(n-2)(n-3) \frac{\left(1-e^{-\lambda_{0} \Delta}\right)^{4}}{\left(\lambda_{0} \Delta \alpha\right)^{4}}+4 n(n-1) \frac{2\left(1-e^{-\lambda_{0} \Delta}\right)\left(1-e^{-3 \lambda_{0} \Delta}\right)}{\left(\lambda_{0} \Delta\right)^{2} \alpha^{4}} \\
& +n \frac{6\left(1-e^{-4 \lambda_{0} \Delta}\right)}{\lambda_{0} \Delta \alpha^{4}}+6 n(n-1)(n-2) \frac{\left(1-e^{-\lambda_{0} \Delta}\right)^{2}\left(1-e^{-2 \lambda_{0} \Delta}\right)}{\left(\lambda_{0} \Delta\right)^{3} \alpha^{4}} \\
& +3 n(n-1) \frac{\left(1-e^{-2 \lambda_{0} \Delta}\right)^{2}}{\left(\lambda_{0} \Delta\right)^{2} \alpha^{4}}
\end{aligned}
$$

其中 $V_{n}=\sum_{i=1}^{n} W_{i}, W_{i}(i=1,2, \cdots)$ 是密度由 (2.7) 式定义的独立同分布随机变 量.

证明 首先, 密度由 (2.7) 式所定义的随机变量 $W_{i}(i=1,2, \cdots)$ 满足

$$
\begin{aligned}
& \mathbb{E}\left|W_{i}\right|=\mathbb{E}\left[W_{1}\right]=\frac{1-e^{-\lambda_{0} \Delta}}{\lambda_{0} \Delta \alpha}, \quad \mathbb{E}\left|W_{i}\right|^{2}=\mathbb{E}\left[W_{1}^{2}\right]=\frac{1-e^{-2 \lambda_{0} \Delta}}{\lambda_{0} \Delta \alpha^{2}}, \\
& \mathbb{E}\left|W_{i}\right|^{3}=\mathbb{E}\left[W_{1}^{3}\right]=\frac{2\left(1-e^{-3 \lambda_{0} \Delta}\right)}{\lambda_{0} \Delta \alpha^{3}}, \quad \mathbb{E}\left|W_{i}\right|^{4}=\mathbb{E}\left[W_{1}^{4}\right]=\frac{6\left(1-e^{-4 \lambda_{0} \Delta}\right)}{\lambda_{0} \Delta \alpha^{4}},
\end{aligned}
$$

因此

$$
\begin{aligned}
\mathbb{E}\left[V_{n}\right] & =n \mathbb{E}\left[W_{1}\right]=n \frac{1-e^{-\lambda_{0} \Delta}}{\lambda_{0} \Delta \alpha} . \\
\mathbb{E}\left[V_{n}^{2}\right] & =\mathbb{E}\left[\sum_{i=1}^{n} W_{i}^{2}+2 \sum_{i=1}^{n} \sum_{j=i+1}^{n} W_{i} W_{j}\right]=\sum_{i=1}^{n} \mathbb{E}\left[W_{i}^{2}\right]+2 \sum_{i=1}^{n} \sum_{j=i+1}^{n} \mathbb{E}\left[W_{i}\right] \mathbb{E}\left[W_{j}\right] \\
& =n \mathbb{E}\left[W_{1}^{2}\right]+n(n-1)\left(\mathbb{E}\left[W_{1}\right]\right)^{2}=n \frac{1-e^{-2 \lambda_{0} \Delta}}{\lambda_{0} \Delta \alpha^{2}}+n(n-1) \frac{\left(1-e^{-\lambda_{0} \Delta}\right)^{2}}{\left(\lambda_{0} \Delta \alpha\right)^{2}}
\end{aligned}
$$

类似的方法亦可证明 (5.11) 和 (5.12) 式.

由 (5.9)-(5.12) 式, 我们得到如下一些不等式:

$$
\begin{aligned}
\mathbb{E}\left[1+e^{\lambda_{0} \Delta} V_{n}\right]= & +n \frac{e^{\lambda_{0} \Delta}-1}{\lambda_{0} \Delta \alpha}, \\
\mathbb{E}\left[\left(1+e^{\lambda_{0} \Delta} V_{n}\right)^{2}\right]=1 & +2 n \frac{e^{\lambda_{0} \Delta}-1}{\lambda_{0} \Delta \alpha}+n \frac{e^{2 \lambda_{0} \Delta}-1}{\lambda_{0} \Delta \alpha^{2}}+n(n-1) \frac{\left(e^{\lambda_{0} \Delta}-1\right)^{2}}{\left(\lambda_{0} \Delta \alpha\right)^{2}},(5 . \\
\mathbb{E}\left[\left(1+e^{\lambda_{0} \Delta} V_{n}\right)^{3}\right]= & +3 n \frac{e^{\lambda_{0} \Delta}-1}{\lambda_{0} \Delta \alpha}+3 n \frac{e^{2 \lambda_{0} \Delta}-1}{\lambda_{0} \Delta \alpha^{2}}+3 n(n-1) \frac{\left(e^{\lambda_{0} \Delta}-1\right)^{2}}{\left(\lambda_{0} \Delta \alpha\right)^{2}} \\
& +2 n \frac{e^{3 \lambda_{0} \Delta}-1}{\lambda_{0} \Delta \alpha^{3}}+3 n(n-1) \frac{\left(e^{\lambda_{0} \Delta}-1\right)^{2}\left(e^{\lambda_{0} \Delta}+1\right)}{\left(\lambda_{0} \Delta\right)^{2} \alpha^{3}} \\
& +n(n-1)(n-2) \frac{\left(e^{\lambda_{0} \Delta}-1\right)^{3}}{\left(\lambda_{0} \Delta \alpha\right)^{3}},
\end{aligned}
$$




$$
\begin{aligned}
& \mathbb{E}\left[\left(1+e^{\lambda_{0} \Delta} V_{n}\right)^{4}\right]=1+4 n \frac{e^{\lambda_{0} \Delta}-1}{\lambda_{0} \Delta \alpha}+6 n \frac{e^{2 \lambda_{0} \Delta}-1}{\lambda_{0} \Delta \alpha^{2}}+6 n(n-1) \frac{\left(e^{\lambda_{0} \Delta}-1\right)^{2}}{\left(\lambda_{0} \Delta \alpha\right)^{2}} \\
& +8 n \frac{e^{3 \lambda_{0} \Delta}-1}{\lambda_{0} \Delta \alpha^{3}}+12 n(n-1) \frac{\left(e^{\lambda_{0} \Delta}-1\right)^{2}\left(e^{\lambda_{0} \Delta}+1\right)}{\left(\lambda_{0} \Delta\right)^{2} \alpha^{3}} \\
& +6 n \frac{e^{4 \lambda_{0} \Delta}-1}{\lambda_{0} \Delta \alpha^{4}}+4 n(n-1)(n-2) \frac{\left(e^{\lambda_{0} \Delta}-1\right)^{3}}{\left(\lambda_{0} \Delta \alpha\right)^{3}} \\
& +8 n(n-1) \frac{\left(e^{\lambda_{0} \Delta}-1\right)\left(e^{3 \lambda_{0} \Delta}-1\right)}{\left(\lambda_{0} \Delta\right)^{2} \alpha^{4}}+3 n(n-1) \frac{\left(e^{2 \lambda_{0} \Delta}-1\right)^{2}}{\left(\lambda_{0} \Delta\right)^{2} \alpha^{4}} \\
& +n(n-1)(n-2)(n-3) \frac{\left(e^{\lambda_{0} \Delta}-1\right)^{4}}{\left(\lambda_{0} \Delta \alpha\right)^{4}} \\
& +6 n(n-1)(n-2) \frac{\left(e^{\lambda_{0} \Delta}-1\right)^{2}\left(e^{2 \lambda_{0} \Delta}-1\right)}{\left(\lambda_{0} \Delta\right)^{3} \alpha^{4}}, \\
& \mathbb{E}\left[1+e^{2 \lambda_{0} \Delta} V_{n}^{2}\right]=1+n \frac{e^{2 \lambda_{0} \Delta}-1}{\lambda_{0} \Delta \alpha^{2}}+n(n-1) \frac{\left(e^{\lambda_{0} \Delta}-1\right)^{2}}{\left(\lambda_{0} \Delta \alpha\right)^{2}}, \\
& \mathbb{E}\left[\left(1+e^{\lambda_{0} \Delta} V_{n}\right)\left(1+e^{2 \lambda_{0} \Delta} V_{n}^{2}\right)\right]=1+n \frac{e^{\lambda_{0} \Delta}-1}{\lambda_{0} \Delta \alpha}+n \frac{e^{2 \lambda_{0} \Delta}-1}{\lambda_{0} \Delta \alpha^{2}}+n(n-1) \frac{\left(e^{\lambda_{0} \Delta}-1\right)^{2}}{\left(\lambda_{0} \Delta \alpha\right)^{2}} \\
& +2 n \frac{e^{3 \lambda_{0} \Delta}-1}{\lambda_{0} \Delta \alpha^{3}}+3 n(n-1) \frac{\left(e^{\lambda_{0} \Delta}-1\right)^{2}\left(e^{\lambda_{0} \Delta}+1\right)}{\left(\lambda_{0} \Delta\right)^{2} \alpha^{3}} \\
& +n(n-1)(n-2) \frac{\left(e^{\lambda_{0} \Delta}-1\right)^{3}}{\left(\lambda_{0} \Delta \alpha\right)^{3}}, \\
& \mathbb{E}\left[\left(1+e^{\lambda_{0} \Delta} V_{n}\right)^{2}\left(1+e^{2 \lambda_{0} \Delta} V_{n}^{2}\right)\right] \\
& =1+2 n \frac{e^{\lambda_{0} \Delta}-1}{\lambda_{0} \Delta \alpha}+2 n \frac{e^{2 \lambda_{0} \Delta}-1}{\lambda_{0} \Delta \alpha^{2}}+2 n(n-1) \frac{\left(e^{\lambda_{0} \Delta}-1\right)^{2}}{\left(\lambda_{0} \Delta \alpha\right)^{2}} \\
& +3 n(n-1) \frac{\left(e^{2 \lambda_{0} \Delta}-1\right)^{2}}{\left(\lambda_{0} \Delta\right)^{2} \alpha^{4}}+6 n(n-1)(n-2) \frac{\left(e^{\lambda_{0} \Delta}-1\right)^{2}\left(e^{2 \lambda_{0} \Delta}-1\right)}{\left(\lambda_{0} \Delta\right)^{3} \alpha^{4}} \\
& +2 n(n-1)(n-2) \frac{\left(e^{\lambda_{0} \Delta}-1\right)^{3}}{\left(\lambda_{0} \Delta \alpha\right)^{3}}+8 n(n-1) \frac{\left(e^{\lambda_{0} \Delta}-1\right)\left(e^{3 \lambda_{0} \Delta}-1\right)}{\left(\lambda_{0} \Delta\right)^{2} \alpha^{4}} \\
& +4 n \frac{e^{3 \lambda_{0} \Delta}-1}{\lambda_{0} \Delta \alpha^{3}}+6 n(n-1) \frac{\left(e^{\lambda_{0} \Delta}-1\right)^{2}\left(e^{\lambda_{0} \Delta}+1\right)}{\left(\lambda_{0} \Delta\right)^{2} \alpha^{3}}+6 n \frac{e^{4 \lambda_{0} \Delta}-1}{\lambda_{0} \Delta \alpha^{4}} \\
& +n(n-1)(n-2)(n-3) \frac{\left(e^{\lambda_{0} \Delta}-1\right)^{4}}{\left(\lambda_{0} \Delta \alpha\right)^{4}} \\
& \mathbb{E}\left[\left(1+e^{2 \lambda_{0} \Delta} V_{n}^{2}\right)^{2}\right]=1+2 n \frac{e^{2 \lambda_{0} \Delta}-1}{\lambda_{0} \Delta \alpha^{2}}+2 n(n-1) \frac{\left(e^{\lambda_{0} \Delta}-1\right)^{2}}{\left(\lambda_{0} \Delta \alpha\right)^{2}}+6 n \frac{e^{4 \lambda_{0} \Delta}-1}{\lambda_{0} \Delta \alpha^{4}} \\
& +8 n(n-1) \frac{\left(e^{\lambda_{0} \Delta}-1\right)\left(e^{3 \lambda_{0} \Delta}-1\right)}{\left(\lambda_{0} \Delta\right)^{2} \alpha^{4}}+3 n(n-1) \frac{\left(e^{2 \lambda_{0} \Delta}-1\right)^{2}}{\left(\lambda_{0} \Delta\right)^{2} \alpha^{4}} \\
& +6 n(n-1)(n-2) \frac{\left(e^{\lambda_{0} \Delta}-1\right)^{2}\left(e^{2 \lambda_{0} \Delta}-1\right)}{\left(\lambda_{0} \Delta\right)^{3} \alpha^{4}} \\
& +n(n-1)(n-2)(n-3) \frac{\left(e^{\lambda_{0} \Delta}-1\right)^{4}}{\left(\lambda_{0} \Delta \alpha\right)^{4}},
\end{aligned}
$$




$$
\begin{aligned}
\mathbb{E}\left[1+e^{3 \lambda_{0} \Delta} V_{n}^{3}\right]= & +2 n \frac{e^{3 \lambda_{0} \Delta}-1}{\lambda_{0} \Delta \alpha^{3}}+3 n(n-1) \frac{\left(e^{\lambda_{0} \Delta}-1\right)^{2}\left(e^{\lambda_{0} \Delta}+1\right)}{\left(\lambda_{0} \Delta\right)^{2} \alpha^{3}} \\
& +n(n-1)(n-2) \frac{\left(e^{\lambda_{0} \Delta}-1\right)^{3}}{\left(\lambda_{0} \Delta \alpha\right)^{3}} .
\end{aligned}
$$

引理 5.3 对于固定的时间间隔 $\Delta>0$,

(i) 当 $c=c_{0}>0, \alpha=\alpha_{0}>0$ 时, $\mathbb{E}\left[\left|\frac{A_{i}}{A_{0}} \mathbb{I}_{\left\{Z_{1}>0\right\}}\right|^{4-i}\right](i=1,2,3)$ 都存在;

(ii) 当 $c=c_{0}>0, \alpha=\alpha_{0}>1$ 时, $\mathbb{E}\left[\left|\frac{C_{i j}}{A_{0}} \mathbb{I}_{\left\{Z_{1}>0\right\}}\right|^{4-(i+j)}\right]((i, j)=(1,1),(1,2)$ 或 $(2,1))$ 和 $\mathbb{E}\left[\left|\frac{B_{i}}{A_{0}} \mathbb{I}_{\left\{Z_{1}>0\right\}}\right|^{4-i}\right](i=1,2)$ 都存在;

(iii) 当 $c=c_{0}>0, \alpha=\alpha_{0}>2^{\frac{2}{3}}$ 时, $\mathbb{E}\left[\left|\frac{B_{3}}{A_{0}} \mathbb{I}_{\left\{Z_{1}>0\right\}}\right|\right]$ 存在.

证明 本节开始时早已证明了引理 (i) 部分. 由 (5.1)-(5.3) 和 (5.13)-(5.21) 式, 用证明引理 (i) 部分相同的方法, 易证引理 (ii) 和 (iii) 部分, 作为例子, 这里 仅证明 $\mathbb{E}\left[\left|\frac{C_{12}}{A_{0}} \mathbb{I}_{\left\{Z_{1}>0\right\}}\right|\right]$ 的存在性.

$$
\begin{aligned}
& \mathbb{E}\left[\left|\frac{C_{12}}{A_{0}} \mathbb{I}_{\left\{Z_{1}>0\right\}}\right|\right] \\
\leqslant & \int_{0}^{\infty} \sum_{n=1}^{\infty} \frac{\left(\lambda_{0} c_{0} \Delta\right)^{n-1}}{(n-1) !}\left|\frac{\partial^{2} f_{n}(x)}{\partial \alpha^{2}}\right| \mathrm{d} x \\
\leqslant & \int_{0}^{\infty} \sum_{n=1}^{\infty} \frac{\left(\lambda_{0} c_{0} \Delta\right)^{n-1}}{(n-1) !}\left[n\left(1+x^{2} e^{2 \lambda_{0} \Delta}\right)+n(n-1)\left(1+x e^{\lambda_{0} \Delta}\right)^{2}\right] f_{n}(x) \mathrm{d} x \\
= & \sum_{n=1}^{\infty} \frac{n\left(\lambda_{0} c_{0} \Delta\right)^{n-1}}{(n-1) !} \mathbb{E}\left[1+e^{2 \lambda_{0} \Delta} V_{n}^{2}\right]+\sum_{n=2}^{\infty} \frac{n\left(\lambda_{0} c_{0} \Delta\right)^{n-1}}{(n-2) !} \mathbb{E}\left[\left(1+e^{\lambda_{0} \Delta} V_{n}\right)^{2}\right] .
\end{aligned}
$$

由最后一个等式右端无穷级数的收玫性, $\mathbb{E}\left[\left|\frac{C_{12}}{A_{0}} \mathbb{I}_{\left\{Z_{1}>0\right\}}\right|\right]<\infty$ 是显然的.

\section{2 相合性及渐近正态性}

从现在开始, 记 $\Theta:=(0, \infty) \times\left(2^{\frac{2}{3}}, \infty\right)$. 在参数 $\lambda$ 估出之后, $(c, \alpha)$ 的最大似 然估计为使其对数似然函数在参数空间内达到最大值的量, 记为 $\left(\hat{c}_{n}, \hat{\alpha}_{n}\right)$, 即

$$
\left(\hat{c}_{n}, \hat{\alpha}_{n}\right)=\arg \max \left\{L_{n}(c, \alpha):(c, \alpha) \in \Theta\right\} .
$$

一般地, 通过取对数似然函数关于 $(c, \alpha)$ 的导数, 并令它们等于 0 , 可得似然方程

$$
\left\{\begin{array}{l}
\frac{\partial L_{n}(c, \alpha)}{\partial c}=\sum_{k=0}^{n} \frac{\partial g}{\partial c}\left(\Delta, x_{k} ; x_{k-1}, \lambda_{0}, c, \alpha\right)=0, \\
\frac{\partial L_{n}(c, \alpha)}{\partial \alpha}=\sum_{k=0}^{n} \frac{\partial g}{\partial \alpha}\left(\Delta, x_{k} ; x_{k-1}, \lambda_{0}, c, \alpha\right)=0,
\end{array}\right.
$$

使得 $L_{n}^{(J)}(c, \alpha)$ 达到最大的参数值 $(c, \alpha)$, 记为 $\left(\hat{c}_{n}^{(J)}, \hat{\alpha}_{n}^{(J)}\right)$, 即

$$
\left(\hat{c}_{n}^{(J)}, \hat{\alpha}_{n}^{(J)}\right)=\arg \max \left\{L_{n}^{(J)}(c, \alpha):(c, \alpha) \in \Theta\right\} .
$$

令 $\left(c_{0}, \alpha_{0}\right)$ 为参数 $(c, \alpha)$ 的真值, 并记

$$
\frac{D g}{D(c, \alpha)}:=\left(\begin{array}{c}
\frac{\partial g}{\partial c} \\
\frac{\partial g}{\partial \alpha}
\end{array}\right) ; \quad \frac{D^{2} g}{D(c, \alpha)^{2}}:=\left(\begin{array}{cc}
\frac{\partial^{2} g}{\partial c^{2}} & \frac{\partial^{2} g}{\partial c \partial \alpha} \\
\frac{\partial^{2} g}{\partial c \partial \alpha} & \frac{\partial^{2} g}{\partial \alpha^{2}}
\end{array}\right) .
$$


为简短起见, 这里省略了变量 $\left(\Delta, x_{k} ; x_{k-1}, \lambda_{0}, c, \alpha\right)$. 以下用 $\mathbb{E}\{f\}$ 作为 $\mathbb{E}\left\{f\left(\Delta, X_{1}\right.\right.$; $\left.\left.X_{0}, \lambda_{0}, c_{0}, \alpha_{0}\right)\right\}$ 的缩写, 其中 $f$ 为变量 $\left(\Delta, X_{1} ; X_{0}, \lambda_{0}, c_{0}, \alpha_{0}\right)$ 的任一函数.

引理 5.4 对于固定的时间间隔 $\Delta>0$, 固定的 $\lambda_{0}>0$ 和 $\left(c_{0}, \alpha_{0}\right) \in \Theta$, 当 $n \rightarrow \infty$ 时,

$$
\begin{gathered}
\frac{1}{n} \sum_{k=1}^{n} \frac{D g}{D(c, \alpha)}\left(\Delta, X_{k} ; X_{k-1}, \lambda_{0}, c_{0}, \alpha_{0}\right) \rightarrow \mathbf{0}_{2 \times 1} \\
\frac{1}{n} \sum_{k=1}^{n} \frac{D^{2} g}{D(c, \alpha)^{2}}\left(\Delta, X_{k} ; X_{k-1}, \lambda_{0}, c_{0}, \alpha_{0}\right) \rightarrow-\boldsymbol{\Sigma}_{2 \times 2}
\end{gathered}
$$

其中

$$
\boldsymbol{\Sigma}_{2 \times 2}=\left(\begin{array}{ll}
\mathbb{E}\left\{\left(\frac{\partial g}{\partial c}\right)^{2}\right\} & \mathbb{E}\left\{\frac{\partial g}{\partial c} \frac{\partial g}{\partial \alpha}\right\} \\
\mathbb{E}\left\{\frac{\partial g}{\partial c} \frac{\partial g}{\partial \alpha}\right\} & \mathbb{E}\left\{\left(\frac{\partial g}{\partial \alpha}\right)^{2}\right\}
\end{array}\right)
$$

是一正定矩阵. 设 $\omega$ 为参数真值 $\left(c_{0}, \alpha_{0}\right)$ 的一个小邻域,

$$
\omega=\left\{(c, \alpha):\left\|(c, \alpha)-\left(c_{0}, \alpha_{0}\right)\right\|<\bar{\delta}, \bar{\delta}>0\right\} .
$$

令

$$
G\left(\Delta, X_{k} ; X_{k-1}, \lambda_{0}, c, \alpha\right)=\sup _{(c, \alpha) \in \omega}\left\{\left|\frac{\partial^{3} g\left(\Delta, X_{k} ; X_{k-1}, \lambda_{0}, c, \alpha\right)}{\partial c^{i} \partial \alpha^{3-i}}\right|, i=0,1,2,3 .\right\},
$$

则

$$
\frac{1}{n} \sum_{k=1}^{n} G\left(\Delta, X_{k} ; X_{k-1}, \lambda_{0}, c, \alpha\right) \rightarrow M \quad \text { a.s. }
$$

其中 $0 \leqslant M<\infty$ 为一常数.

证明 由引理 5.3, 该引理中所涉及的数学期望都是有意义的. 首先, 对固 定的 $\Delta>0$,

$$
\begin{aligned}
\mathbb{E}\left\{\frac{\partial g}{\partial c}\right\} & =-\lambda_{0} \Delta+\mathbb{E}\left\{\frac{\partial h}{\partial c} \mathbb{I}_{\left\{X_{1}>e^{-\lambda_{0} \Delta} X_{0}\right\}}\right\} \\
& =-\lambda_{0} \Delta+\mathbb{E}\left\{\mathbb{E}\left[\frac{\partial h}{\partial c} \mathbb{I}_{\left\{X_{1}>e^{-\lambda_{0} \Delta} X_{0}\right\}} \mid X_{0}\right]\right\} \\
& =-\lambda_{0} \Delta+e^{-\lambda_{0} c_{0} \Delta} \mathbb{E}\left\{\left.\int_{e^{-\lambda_{0} \Delta} X_{0}}^{\infty} \frac{\partial p\left(y-e^{-\lambda_{0} \Delta} X_{0}\right)}{\partial c}\right|_{\substack{c=c_{0} \\
\alpha=\alpha_{0}}} \mathrm{~d} y\right\} \\
& =-\lambda_{0} \Delta+e^{-\lambda_{0} c_{0} \Delta} \mathbb{E}\left\{\left.\frac{\partial}{\partial c} \int_{e^{-\lambda_{0} \Delta} X_{0}}^{\infty} p\left(y-e^{-\lambda_{0} \Delta} X_{0}\right) \mathrm{d} y\right|_{\substack{c=c_{0} \\
\alpha=\alpha_{0}}}\right\} \\
& =-\lambda_{0} \Delta+e^{-\lambda_{0} c_{0} \Delta} \mathbb{E}\left\{\left.\frac{\partial}{\partial c}\left(e^{\lambda_{0} c \Delta}-1\right)\right|_{\substack{c=c_{0} \\
\alpha=\alpha_{0}}}\right\} \\
& =-\lambda_{0} \Delta+e^{-\lambda_{0} c_{0} \Delta} e^{\lambda_{0} c_{0} \Delta} \lambda_{0} \Delta=0,
\end{aligned}
$$

其中 $p(x)$ 由 (2.14) 式定义, 对 $\mathbb{E}\left\{\frac{\partial g}{\partial c}\right\}$ 进行类似的处理, 可以得到 $\mathbb{E}\left\{\frac{\partial g}{\partial \alpha}\right\}=0$, 亦 
即 $\mathbb{E}\left\{\frac{D g}{D(c, \alpha)}\left(\Delta, X_{1} ; X_{0}, \lambda_{0}, c_{0}, \alpha_{0}\right)\right\}=\mathbf{0}_{\mathbf{2} \times \mathbf{1}}$. 其次, 对固定的 $\Delta>0$,

$$
\begin{aligned}
\mathbb{E}\left\{\frac{\partial^{2} g}{\partial c^{2}}\right\}= & \mathbb{E}\left\{\mathbb{E}\left[\left.\frac{\partial^{2}}{\partial c^{2}}\left(-\lambda_{0} c \Delta+h\left(\Delta, X_{1} ; X_{0}, \lambda_{0}, c, \alpha\right) \mathbb{I}_{\left\{X_{1}>e^{-\lambda_{0} \Delta} X_{0}\right\}}\right)\right|_{\substack{c=c_{0} \\
\alpha=\alpha_{0}}} \mid X_{0}\right]\right\} \\
= & \mathbb{E}\left\{\mathbb{E}\left[\frac{\partial^{2}}{\partial c^{2}} h\left(\Delta, X_{1} ; X_{0}, \lambda_{0}, c_{0}, \alpha_{0}\right) \mathbb{I}_{\left.\left\{X_{1}>e^{-\lambda_{0} X_{X_{0}}} \mid X_{0}\right]\right\}}\right]\right. \\
= & e^{-\lambda_{0} c_{0} \Delta} \mathbb{E}\left\{\left.\frac{\partial^{2}}{\partial c^{2}} \int_{e^{-\lambda_{0} \Delta} X_{0}}^{\infty} p\left(y-e^{-\lambda_{0} \Delta} X_{0}\right) \mathrm{d} y\right|_{\substack{c=c_{0} \\
\alpha=\alpha_{0}}}\right\} \\
& -\mathbb{E}\left\{\mathbb{E}\left[\left(\lambda_{0} \Delta+\frac{\partial g}{\partial c}\right)^{2} \mid X_{0}\right]\right\} \\
= & e^{-\lambda_{0} c_{0} \Delta} \mathbb{E}\left\{\left.\frac{\partial^{2}}{\partial c^{2}}\left[e^{\lambda_{0} c \Delta}\left(1-e^{-\lambda_{0} c \Delta}\right)\right]\right|_{\substack{c=c_{0} \\
\alpha=\alpha_{0}}}\right\}-\left(\lambda_{0} \Delta\right)^{2}-\mathbb{E}\left\{\left(\frac{\partial g}{\partial c}\right)^{2}\right\} \\
= & -\mathbb{E}\left\{\left(\frac{\partial g}{\partial c}\right)^{2}\right\} .
\end{aligned}
$$

用类似的证明过程，以下等式也是成立的:

$$
\mathbb{E}\left\{\frac{\partial^{2} g}{\partial c \partial \alpha}\right\}=-\mathbb{E}\left\{\frac{\partial g}{\partial c} \frac{\partial g}{\partial \alpha}\right\}, \quad \mathbb{E}\left\{\frac{\partial^{2} g}{\partial \alpha^{2}}\right\}=-\mathbb{E}\left\{\left(\frac{\partial g}{\partial \alpha}\right)^{2}\right\} .
$$

亦即 $\mathbb{E}\left\{\frac{D^{2} g}{D(c, \alpha)^{2}}\left(\Delta, X_{1} ; X_{0}, \lambda_{0}, c_{0}, \alpha_{0}\right)\right\}=-\boldsymbol{\Sigma}_{\mathbf{2} \times \mathbf{2}}$. 最后, 由遍历定理 (参见文献 [18]) 及前面的讨论，(5.25) 和 (5.26) 及 (5.28) 式都是成立的.

显然, $\mathbb{E}\left\{\left(\frac{\partial g}{\partial c}\right)^{2}\right\}$ 和 $\mathbb{E}\left\{\left(\frac{\partial g}{\partial \alpha}\right)^{2}\right\}$ 都是正的. 由 Cauchy-Schwarz 不等式 (参见文 献 [19]),

$$
\mathbb{E}\left\{\left(\frac{\partial g}{\partial c}\right)^{2}\right\} \mathbb{E}\left\{\left(\frac{\partial g}{\partial \alpha}\right)^{2}\right\} \geqslant\left(\mathbb{E}\left\{\frac{\partial g}{\partial c} \frac{\partial g}{\partial \alpha}\right\}\right)^{2}
$$

“=” 是不可能的, 因为由 $\mathbb{P}\left(X_{1}>e^{-\lambda_{0} \Delta} X_{0}\right)<1$ 和 Bayes 公式, $\forall a \neq 0$,

$$
\mathbb{P}\left(\frac{\partial g}{\partial c}\left(\Delta, X_{1} ; X_{0}, \lambda_{0}, c_{0}, \alpha_{0}\right)=a \frac{\partial g}{\partial \alpha}\left(\Delta, X_{1} ; X_{0}, \lambda_{0}, c_{0}, \alpha_{0}\right)\right)<1,
$$

所以 $\boldsymbol{\Sigma}_{\mathbf{2} \times \mathbf{2}}$ 为正定矩阵.

记

$$
\frac{D L_{n}}{D(c, \alpha)}(c, \alpha)=\left(\begin{array}{c}
\frac{\partial}{\partial c} L_{n}(c, \alpha) \\
\frac{\partial}{\partial \alpha} L_{n}(c, \alpha)
\end{array}\right)
$$

定理 5.1 在引理 5.4 的条件下, 由 (5.22) 式定义、并由 (5.23) 式计算的最大 似然估计 $\left(\hat{c}_{n}, \hat{\alpha}_{n}\right)$ 关于参数真值 $\left(c_{0}, \alpha_{0}\right)$ 是强相合的.

证明 首先, 将

$$
\frac{D L_{n}}{D(c, \alpha)}(c, \alpha)=\sum_{k=1}^{n} \frac{D g}{D(c, \alpha)}\left(\Delta, X_{k} ; X_{k-1}, \lambda_{0}, c, \alpha\right)
$$


在参数真值 $\left(c_{0}, \alpha_{0}\right)$ 点 Taloy 展开. 由中值定理, 若 $(c, \alpha) \in \omega$, 则

$$
\begin{aligned}
\frac{D L_{n}}{D(c, \alpha)}(c, \alpha)= & \sum_{k=1}^{n} \frac{D g}{D(c, \alpha)}\left(\Delta, X_{k} ; X_{k-1}, \lambda_{0}, c_{0}, \alpha_{0}\right) \\
& +\sum_{k=1}^{n} \frac{D^{2} g}{D(c, \alpha)^{2}}\left(\Delta, X_{k} ; X_{k-1}, \lambda_{0}, c_{0}, \alpha_{0}\right)\left(\begin{array}{c}
c-c_{0} \\
\alpha-\alpha_{0}
\end{array}\right) \\
& +\left\|(c, \alpha)-\left(c_{0}, \alpha_{0}\right)\right\|^{2}\left[\sum_{k=1}^{n} G\left(\Delta, X_{k} ; X_{k-1}, \lambda_{0}, c_{0}, \alpha_{0}\right)\right] \mathbf{M}_{\mathbf{2} \times \mathbf{1}}
\end{aligned}
$$

其中 $\mathbf{M}_{2 \times 1}=\left(\begin{array}{c}m_{1} \\ m_{2}\end{array}\right)$, 且对 $j=1,2,\left|m_{j}\right| \leqslant 2$.

因为 $\boldsymbol{\Sigma}_{\mathbf{2} \times \mathbf{2}}$ 的所有特征值都大于 0 , 所以存在非随机函数 $\lambda\left(c_{0}, \alpha_{0}\right)>0$, 使得

$$
\mathbf{z}^{\mathbf{T}} \mathbf{\Sigma} \mathbf{z} \geqslant \lambda\left(c_{0}, \alpha_{0}\right)\|\mathbf{z}\|, \quad\|\mathbf{z}\|=\mathbf{z}^{T} \mathbf{z} .
$$

由 (5.29)、 (5.25)、(5.26) 和 (5.28) 式, 若 $\left\|(c, \alpha)-\left(c_{0}, \alpha_{0}\right)\right\|<\bar{\delta}$, 则有

$$
\begin{aligned}
\lim _{n \rightarrow \infty}\left|\frac{1}{n} \sum_{k=1}^{n} \frac{\partial g}{\partial c}\left(\Delta, X_{k} ; X_{k-1}, \lambda_{0}, c, \alpha\right)+\mathbb{E}\left\{\left(\frac{\partial g}{\partial c}\right)^{2}\right\}\left(c-c_{0}\right)+\mathbb{E}\left\{\frac{\partial g}{\partial c} \frac{\partial g}{\partial \alpha}\right\}\left(\alpha-\alpha_{0}\right)\right| \\
=\lim _{n \rightarrow \infty} \mid \frac{1}{n} \sum_{k=1}^{n} \frac{\partial g}{\partial c}\left(\Delta, X_{k} ; X_{k-1}, \lambda_{0}, c_{0}, \alpha_{0}\right) \\
+\left\{\frac{1}{n} \sum_{k=1}^{n} \frac{\partial^{2} g}{\partial c^{2}}\left(\Delta, X_{k} ; X_{k-1}, \lambda_{0}, c_{0}, \alpha_{0}\right)+\mathbb{E}\left\{\left(\frac{\partial g}{\partial c}\right)^{2}\right\}\right\}\left(c-c_{0}\right) \\
\quad+\left\{\frac{1}{n} \sum_{k=1}^{n} \frac{\partial^{2} g}{\partial c \partial \alpha}\left(\Delta, X_{k} ; X_{k-1}, \lambda_{0}, c_{0}, \alpha_{0}\right)+\mathbb{E}\left\{\frac{\partial g}{\partial c} \frac{\partial g}{\partial \alpha}\right\}\right\}\left(\alpha-\alpha_{0}\right) \\
\quad+m_{1}\left\|(c, \alpha)-\left(c_{0}, \alpha_{0}\right)\right\|^{2}\left[\frac{1}{n} \sum_{k=1}^{n} G\left(\Delta, X_{k} ; X_{k-1}, \lambda_{0}, c_{0}, \alpha_{0}\right)\right] \mid \\
\leqslant \lim _{n \rightarrow \infty}\left|\frac{1}{n} \sum_{k=1}^{n} \frac{\partial g}{\partial c}\left(\Delta, X_{k} ; X_{k-1}, \lambda_{0}, c_{0}, \alpha_{0}\right)\right| \\
+\lim _{n \rightarrow \infty}\left|\frac{1}{n} \sum_{k=1}^{n} \frac{\partial^{2} g}{\partial c^{2}}\left(\Delta, X_{k} ; X_{k-1}, \lambda_{0}, c_{0}, \alpha_{0}\right)+\mathbb{E}\left\{\left(\frac{\partial g}{\partial c}\right)^{2}\right\}\right|\left|c-c_{0}\right| \\
\quad+\lim _{n \rightarrow \infty}\left|\frac{1}{n} \sum_{k=1}^{n} \frac{\partial^{2} g}{\partial c \partial \alpha}\left(\Delta, X_{k} ; X_{k-1}, \lambda_{0}, c_{0}, \alpha_{0}\right)+\mathbb{E}\left\{\frac{\partial g}{\partial c} \frac{\partial g}{\partial \alpha}\right\}\right|\left|\alpha-\alpha_{0}\right| \\
+2 \lim _{n \rightarrow \infty}\left|\left[\frac{1}{n} \sum_{k=1}^{n} G\left(\Delta, X_{k} ; X_{k-1}, \lambda_{0}, c_{0}, \alpha_{0}\right)\right]\right|\left\|(c, \alpha)-\left(c_{0}, \alpha_{0}\right)\right\|^{2} \\
=2 M\left\|(c, \alpha)-\left(c_{0}, \alpha_{0}\right)\right\|^{2}, \quad \text { a.s. },
\end{aligned}
$$

同理可得

$$
\lim _{n \rightarrow \infty} \mid \frac{1}{n} \sum_{k=1}^{n} \frac{\partial g}{\partial \alpha}\left(\Delta, X_{k} ; X_{k-1}, \lambda_{0}, c, \alpha\right)
$$




$$
\begin{aligned}
& +\mathbb{E}\left\{\frac{\partial g}{\partial c} \frac{\partial g}{\partial \alpha}\right\}\left(c-c_{0}\right)+\mathbb{E}\left\{\left(\frac{\partial g}{\partial \alpha}\right)^{2}\right\}\left(\alpha-\alpha_{0}\right) \mid \\
\leqslant & 2 M\left\|(c, \alpha)-\left(c_{0}, \alpha_{0}\right)\right\|^{2}, \quad \text { a.s. },
\end{aligned}
$$

对任一 $\varepsilon>0$, 可以选择充分小的 $\delta=\delta(\varepsilon)>0$, 使得

$$
\delta<\min \left\{\varepsilon, \bar{\delta}, \frac{\lambda\left(c_{0}, \alpha_{0}\right)}{4 M}\right\} .
$$

由 (5.30) 式, 若 $\left\|(c, \alpha)-\left(c_{0}, \alpha_{0}\right)\right\|=\delta$, 则

$$
\begin{aligned}
& \lim _{n \rightarrow \infty} \frac{1}{n} \sum_{k=1}^{n}\left(\begin{array}{c}
\frac{\partial g}{\partial c}\left(\Delta, X_{k} ; X_{k-1}, \lambda_{0}, c, \alpha\right) \\
\frac{\partial g}{\partial \alpha}\left(\Delta, X_{k} ; X_{k-1}, \lambda_{0}, c, \alpha\right)
\end{array}\right)^{T}\left(\begin{array}{c}
c-c_{0} \\
\alpha-\alpha_{0}
\end{array}\right) \\
& \leqslant-\left(c-c_{0}, \quad \alpha-\alpha_{0}\right) \boldsymbol{\Sigma}_{\mathbf{2} \times \mathbf{2}}\left(\begin{array}{c}
c-c_{0} \\
\alpha-\alpha_{0}
\end{array}\right)+4 M\left\|(c, \alpha)-\left(c_{0}, \alpha_{0}\right)\right\|^{3} \\
& \leqslant\left(4 M \delta-\lambda\left(c_{0}, \alpha_{0}\right)\right) \delta^{2}<0, \quad \text { a.s., }
\end{aligned}
$$

除掉一个零测集, 我们可以选择 $n_{0}(\varepsilon)$ 充分大, 使得当 $n \geqslant n_{0}(\varepsilon)$ 时,

$$
\frac{1}{n} \sum_{k=1}^{n}\left(\begin{array}{l}
\frac{\partial g}{\partial c}\left(\Delta, X_{k} ; X_{k-1}, \lambda_{0}, c, \alpha\right) \\
\frac{\partial g}{\partial \alpha}\left(\Delta, X_{k} ; X_{k-1}, \lambda_{0}, c, \alpha\right)
\end{array}\right)^{T}\left(\begin{array}{c}
c-c_{0} \\
\alpha-\alpha_{0}
\end{array}\right)<0 .
$$

据文献 [20] 中的引理 2, 存在值 $\left(\hat{c}_{n}, \hat{\alpha}_{n}\right)$, 使得 $\left\|\left(\hat{c}_{n}, \hat{\alpha}_{n}\right)-\left(c_{0}, \alpha_{0}\right)\right\|<\delta<\varepsilon$ 且

$$
\frac{1}{n} \sum_{k=1}^{n}\left(\begin{array}{l}
\frac{\partial g}{\partial c}\left(\Delta, X_{k} ; X_{k-1}, \lambda_{0}, \hat{c}_{n}, \hat{\alpha}_{n}\right) \\
\frac{\partial g}{\partial \alpha}\left(\Delta, X_{k} ; X_{k-1}, \lambda_{0}, \hat{c}_{n}, \hat{\alpha}_{n}\right)
\end{array}\right)=0,
$$

即 $\left(\hat{c}_{n}, \hat{\alpha}_{n}\right)$ 满足 $(5.23)$ 式. 定理得证.

定理 5.2 在引理 5.4 的条件下, 由 (5.22) 式所定义, 并由 (5.23) 式计算的最 大似然估计是渐近正态的. 即

$$
n^{\frac{1}{2}}\left(\left(\begin{array}{c}
\hat{c}_{n} \\
\hat{\alpha}_{n}
\end{array}\right)-\left(\begin{array}{c}
c_{0} \\
\alpha_{0}
\end{array}\right)\right) \stackrel{d}{\longrightarrow} N\left(\mathbf{0}_{\mathbf{2} \times \mathbf{1}}, \boldsymbol{\Sigma}_{\mathbf{2} \times \mathbf{2}}^{-\mathbf{1}}\right),
$$

其中 $\boldsymbol{\Sigma}_{\mathbf{2} \times \mathbf{2}}$ 由 (5.27) 式定义.

证明 由引理 5.3 和等式 (4.7), 文献 [21, 22] 中的渐近正态性条件满足, 故 (5.31) 式成立.

定理 5.3 在引理 5.4 的条件下,

(i) 对固定的充分大的 $n$, 当 $J \rightarrow \infty$ 时,

$$
\left(\hat{c}_{n}^{(J)}, \hat{\alpha}_{n}^{(J)}\right) \stackrel{p}{\longrightarrow}\left(\hat{c}_{n}, \hat{\alpha}_{n}\right) ;
$$

(ii) 当 $n \rightarrow \infty$ 时, 可选择 $J_{n} \rightarrow \infty$ 充分大, 且满足

$$
\left(\hat{c}_{n}^{\left(J_{n}\right)}, \hat{\alpha}_{n}^{\left(J_{n}\right)}\right) \stackrel{p}{\longrightarrow}\left(c_{0}, \alpha_{0}\right) .
$$

而且可以选择 $\left(\hat{c}_{n}^{\left(J_{n}\right)}, \hat{\alpha}_{n}^{\left(J_{n}\right)}\right)$, 使其同 $\left(\hat{c}_{n}, \hat{\alpha}_{n}\right)$ 一样, 具有定理 5.2 所描述的渐近性 质. 
证明 该定理的证明可分为 4 步.

第 1 步. 对固定的 $t, y, x$ 和 $\lambda, p(t, y ; x, \lambda, c, \alpha)$ 关于 $(c, \alpha) \in(0, \infty) \times(0, \infty)$ 是 连续的, 所以 $L_{n}(c, \alpha)$ 关于 $(c, \alpha) \in(0, \infty) \times(0, \infty)$ 也是连续的. $L_{n}^{(J)}(c, \alpha)$ 关于 $(c, \alpha) \in(0, \infty) \times(0, \infty)$ 的连续性是显然的.

第 2 步. 令

$$
\begin{gathered}
r^{(J)}\left(\Delta, X_{k} ; X_{k-1}, \lambda_{0}, c, \alpha\right):=p\left(\Delta, X_{k} ; X_{k-1}, \lambda_{0}, c, \alpha\right)-p^{(J)}\left(\Delta, X_{k} ; X_{k-1}, \lambda_{0}, c, \alpha\right), \\
R^{(J)}\left(\Delta, X_{k} ; X_{k-1}, \lambda_{0}, c, \alpha\right):=\sup \left\{\left|r^{(J)}\left(\Delta, X_{k} ; X_{k-1}, \lambda_{0}, c, \alpha\right)\right|:(c, \alpha) \in \Theta\right\} .
\end{gathered}
$$

这里 $r^{(J)}\left(\Delta, X_{k} ; X_{k-1}, \lambda_{0}, c, \alpha\right)$ 和 $R^{(J)}\left(\Delta, X_{k} ; X_{k-1}, \lambda_{0}, c, \alpha\right)$ 都是由 $Z_{k}=X_{k}-$ $e^{-\lambda \Delta} X_{k-1}$ 所决定的, 因此我们分别记其为 $r^{(J)}\left(Z_{k} ; c, \alpha\right)$ 和 $R^{(J)}\left(Z_{k} ; c, \alpha\right)$. 由命 题 2.1 和 (2.21) 式, $R^{(J)}\left(Z_{k} ; c, \alpha\right) \leqslant \frac{\ln 2}{Z_{k}} \mathbb{I}_{\left\{Z_{k}>0\right\}} \frac{1}{J !}+\left|o\left(\frac{1}{J !}\right)\right|$, 因此 $\forall \delta>0$ 及 $\forall \varepsilon>0$, 可以选择 $J_{0}>0$, 使得 $J_{0} !>\max \left\{\frac{2 \ln 2\left(e^{\lambda_{0} \Delta}-1\right) \alpha_{0}}{\lambda_{0} \Delta \varepsilon \delta}, \frac{2}{\varepsilon}\right\}$, 则由 (2.10) 和 (2.11) 式, $\forall J>J_{0}$,

$$
\begin{aligned}
\mathbb{P}\left(R^{(J)}\left(Z_{k} ; c, \alpha\right)>\varepsilon\right) & \leqslant \mathbb{P}\left(\frac{\ln 2}{Z_{k}} \mathbb{I}_{\left\{Z_{k}>0\right\}} \frac{1}{J !}>\frac{\varepsilon}{2}\right)=\mathbb{P}\left(0<Z_{k}<\frac{2 \ln 2}{\varepsilon J !}\right) \\
& =\sum_{n=1}^{\infty} \frac{\left(\lambda_{0} c_{0} \Delta\right)^{n} e^{-\lambda_{0} c_{0} \Delta}}{n !} \int_{0}^{\frac{2 \ln 2}{\varepsilon J !}} f_{n}(x) \mathrm{d} x \\
& \leqslant \frac{e^{\lambda_{0} \Delta}-1}{\lambda_{0} \Delta} \alpha_{0} \frac{2 \ln 2}{\varepsilon J_{0} !}<\delta .
\end{aligned}
$$

亦即 $\lim _{J \rightarrow \infty} \mathbb{P}\left(R^{(J)}\left(Z_{k} ; c, \alpha\right)>\varepsilon\right)=0$. 即当 $J \rightarrow \infty$ 时,

$$
R^{(J)}\left(\Delta, X_{k} ; X_{k-1}, \lambda_{0}, c, \alpha\right) \stackrel{p}{\longrightarrow} 0 .
$$

第 3 步. 由第 2 步的证明, $p^{(J)}\left(\Delta, X_{k} ; X_{k-1}, \lambda_{0}, c, \alpha\right) \stackrel{p}{\longrightarrow} p\left(\Delta, X_{k} ; X_{k-1}, \lambda_{0}, c\right.$, $\alpha)(J \rightarrow \infty)$. 据对数函数的连续性,

$$
\ln p^{(J)}\left(\Delta, X_{k} ; X_{k-1}, \lambda_{0}, c, \alpha\right) \stackrel{p}{\longrightarrow} \ln p\left(\Delta, X_{k} ; X_{k-1}, \lambda_{0}, c, \alpha\right)(J \rightarrow \infty) .
$$

因此, 给定充分大的 $n, L_{n}^{(J)}(c, \alpha) \stackrel{p}{\longrightarrow} L_{n}(c, \alpha)(J \rightarrow \infty)$ 关于 $(c, \alpha)$ 是一致的. 既 然对所有的 $n$ 和 $J, L_{n}^{(J)}(c, \alpha), L_{n}(c, \alpha)$ 和它们的导数关于 $(c, \alpha)$ 都是连续的, 所 以当 $J \rightarrow \infty$ 时, $\left(\hat{c}_{n}^{(J)}, \hat{\alpha}_{n}^{(J)}\right) \stackrel{p}{\longrightarrow}\left(\hat{c}_{n}, \hat{\alpha}_{n}\right)$.

第 4 步. 根据定理 (i) 部分的结论, 对于充分大的 $n$, 可以选择 $J_{n}$, 使得 $\left\|\left(\hat{c}_{n}^{\left(J_{n}\right)}, \hat{\alpha}_{n}^{\left(J_{n}\right)}\right)-\left(\hat{c}_{n}, \hat{\alpha}_{n}\right)\right\|$ 依概率充分小. 对每个不同的 $n$, 可以选择 $\left\{J_{n}\right\}$, 使得 $J_{n+1}>J_{n}+1$ 且 $n^{\frac{1}{2}} \Sigma^{\frac{1}{2}}\left(\left(\hat{c}_{n}^{\left(J_{n}\right)}, \hat{\alpha}_{n}^{\left(J_{n}\right)}\right)^{\mathrm{T}}-\left(\hat{c}_{n}, \hat{\alpha}_{n}\right)^{\mathrm{T}}\right)=o_{p}(1)$. 因此, 当 $n \rightarrow \infty$ 时, $J_{n} \rightarrow \infty$ 且依概率有 $\left(\hat{c}_{n}^{\left(J_{n}\right)}, \hat{\alpha}_{n}^{\left(J_{n}\right)}\right) \longrightarrow\left(c_{0}, \alpha_{0}\right)$. 渐近正态性显然成立.

\section{3 模拟实验}

定理 5.3 给出了一种在估出参数 $\lambda$ 后, 得到参数 $(c, \alpha)$ 最大似然估计近似值 的方法. 在此, 我们做了一些模拟实验. 所有的实验都用了 $\mathrm{R}$ 语言中 “stats4”包的 “mle” 函数及其 “L-BFGS-B” 最优化方法. 对每种情形，我们都做了 400 次模拟， 每次模拟都固定 $J=7$ 和下界 $(0.0001,1.5875)$, 样本容量分为 $n=200$ 和 $n=500$ 
两种情形. 我们在表 4 和表 5 中采用 $(5,5)$ 作为最优化初值, 在表 6 中采用 $(4,6)$ 作为最优化初值.

表 4 对不同的 $n$ 和 $\lambda$ 所得的 $(c, \alpha)$ 最大似然估计近似值

\begin{tabular}{|c|c|c|c|c|c|c|c|c|}
\hline \multicolumn{3}{|c|}{ 真值 } & \multicolumn{3}{|c|}{$n=100$} & \multicolumn{3}{|c|}{$n=200$} \\
\hline$\lambda$ & $c$ & $\alpha$ & & Mean & RMSE & & Mean & RMSE \\
\hline \multirow[b]{2}{*}{0.1} & \multirow[b]{2}{*}{3} & \multirow[b]{2}{*}{6} & $c$ & 3.9919 & 0.5303 & $c$ & 4.0205 & 0.4100 \\
\hline & & & $\alpha$ & 6.0893 & 0.8110 & $\alpha$ & 6.0104 & 0.5232 \\
\hline \multirow[b]{2}{*}{0.4} & \multirow{2}{*}{3} & \multirow{2}{*}{6} & $c$ & 3.9705 & 0.3148 & $c$ & 3.9889 & 0.1885 \\
\hline & & & $\alpha$ & 5.9978 & 0.4796 & $\alpha$ & 6.0144 & 0.3145 \\
\hline \multirow[b]{2}{*}{0.8} & \multirow[b]{2}{*}{3} & \multirow[b]{2}{*}{6} & $c$ & 3.9459 & 0.3354 & $c$ & 3.9645 & 0.2094 \\
\hline & & & $\alpha$ & 5.9206 & 0.5226 & $\alpha$ & 5.9530 & 0.3232 \\
\hline \multirow{2}{*}{1} & \multirow{2}{*}{3} & \multirow{2}{*}{6} & $c$ & 3.9362 & 0.3521 & $c$ & 3.9413 & 0.2360 \\
\hline & & & $\alpha$ & 5.9171 & 0.5218 & $\alpha$ & 5.9299 & 0.3668 \\
\hline
\end{tabular}

表 5 对不同的 $n \& c$ 所得的 $(c, \alpha)$ 最大似然估计近似值

\begin{tabular}{|c|c|c|c|c|c|c|c|c|}
\hline \multicolumn{3}{|c|}{ 真值 } & \multicolumn{3}{|c|}{$n=100$} & \multicolumn{3}{|c|}{$n=200$} \\
\hline$\lambda$ & $c$ & $\alpha$ & & Mean & RMSE & & Mean & RMSE \\
\hline \multirow[b]{2}{*}{0.6} & \multirow[b]{2}{*}{3} & \multirow[b]{2}{*}{6} & $c$ & 2.9900 & 0.2488 & $c$ & 2.9816 & 0.1678 \\
\hline & & & $\alpha$ & 6.0128 & 0.5109 & $\alpha$ & 5.9679 & 0.3929 \\
\hline \multirow[b]{2}{*}{0.6} & \multirow[b]{2}{*}{5} & \multirow[b]{2}{*}{6} & $c$ & 4.9081 & 0.4147 & $c$ & 4.9606 & 0.2777 \\
\hline & & & $\alpha$ & 5.9263 & 0.4859 & $\alpha$ & 5.9565 & 0.3213 \\
\hline \multirow[b]{2}{*}{0.6} & \multirow[b]{2}{*}{6} & \multirow[b]{2}{*}{6} & $c$ & 5.8430 & 0.5123 & $c$ & 5.9305 & 0.3420 \\
\hline & & & $\alpha$ & 5.8587 & 0.5393 & $\alpha$ & 5.9241 & 0.3541 \\
\hline \multirow{2}{*}{0.6} & \multirow{2}{*}{8} & \multirow{2}{*}{6} & $c$ & 7.7326 & 0.7250 & $c$ & 7.7609 & 0.5008 \\
\hline & & & $\alpha$ & 5.7940 & 0.5592 & $\alpha$ & 5.8040 & 0.3971 \\
\hline
\end{tabular}

表 6 对不同的 $n \& \alpha$ 所得的 $(c, \alpha)$ 最大似然估计近似值

\begin{tabular}{|c|c|c|c|c|c|c|c|c|}
\hline \multicolumn{3}{|c|}{ 真值 } & \multicolumn{3}{|c|}{$n=100$} & \multicolumn{3}{|c|}{$n=200$} \\
\hline$\lambda$ & $c$ & $\alpha$ & & Mean & RMSE & & Mean & RMSE \\
\hline \multirow[b]{2}{*}{0.6} & \multirow[b]{2}{*}{3} & \multirow[b]{2}{*}{3} & $c$ & 3.0047 & 0.2538 & $c$ & 2.9976 & 0.1484 \\
\hline & & & $\alpha$ & 3.0114 & 0.2607 & $\alpha$ & 3.0092 & 0.1514 \\
\hline \multirow{2}{*}{0.6} & \multirow{2}{*}{3} & \multirow{2}{*}{6} & $c$ & 2.9728 & 0.2494 & $c$ & 2.9876 & 0.1694 \\
\hline & & & $\alpha$ & 5.9973 & 0.5006 & $\alpha$ & 6.0096 & 0.3334 \\
\hline \multirow{2}{*}{0.6} & \multirow{2}{*}{3} & \multirow{2}{*}{12} & $c$ & 2.9774 & 0.2439 & $c$ & 2.9943 & 0.1618 \\
\hline & & & $\alpha$ & 12.0460 & 1.0272 & $\alpha$ & 11.9695 & 0.6162 \\
\hline
\end{tabular}




\section{6 结论}

本文研究了 Gamma-OU 过程的转移函数, 其方法可以为分析其他复合 Poisson 驱动 $\mathrm{OU}$ 过程的转移函数所借鉴. 利用转移密度的 Laplace 变换, 我们给出了 用 Gaver-Stehfest 算法所得的转移密度的近似. 在给定 Gamma-OU 过程较具体的 结构 (见 (3.3) 式) 之后, 首先给出了强度参数 $\lambda$ 的一个估计量, 它具有较弱相合 性更强的性质. 在给定参数 $\lambda$ 的估计之后, 我们建立了其他两参数 $c$ 和 $\alpha$ 的似然 函数. 接着证明了 $(c, \alpha)$ 最大似然估计的相合性及渐近正态性. 最大似然估计的 近似也具有最大似然估计所具有的渐近性质. 蒙特卡罗实验显示, 最大化近似 对数似然函数得到的参数估计非常接近 $(c, \alpha)$ 的真值.

将我们的方法应用于分析金融资产的波动率将在以后的工作中加以讨论. 文中方法还具有广义化至其它类 OU 型过程, 尤其是复合 Poisson 驱动 OU 过程 的可能性.

\section{参考文献}

1 Barndorff-Nielsen O E, Shephard N. Non-Gaussian Ornstein-Uhlenbeck-based models and some of their uses in financial economics (with discussion). J R Statist Soc B, 2001, 63: 167-241

2 Barndorff-Nielsen O E, Shephard N. Modelling by Lévy processes for financial econometrics. In: BarndorffNielsen O E, Mikosch T, Resnick S, eds. Lévy Processes-Theory and Applications. Boston: Birkhauser, 2001, 283-318

3 Lo A W, Maximum likelihood estimation of generalized Itô processes with discretely sampled data. Econometric Theory, 1988, 4: 231-548

4 Petersen A R. A new approach to maximum-likelihood estimation for stochastic differential equations based on discrete observations. Scandinavian Journal of Statistics, 1995, 22: 55-71

5 Santa-Clara P. Simulated likelihood estimation of diffusion with an application to the short term interst rate. Mimeo, UCLA, 1995

6 Ait-Sahalia Y. Maximum likelihood estimation of discretely sampled diffusions: a closed-form approximation approach. Econometrica, 2002, 70: 223-262

7 Egorov A V, Li H, Xu Y. Maximum likelihood estimation of time-inhomogeneous diffusions. Journal of Econometrics, 2003, 114: 107-139

8 Barndorff-Nielsen O E, Shephard N, Econometric analysis of realised volatility and its use in estimating stochastic volatility models. J R Statist Soc B (Statistical Methodology), 2002, 64: 253-280

9 Roberts G O, Papaspiliopoulos O. Bayesian inference for non-Gaussian Ornstein-Uhlenbeck stochastic volatility processes. J R Statist Soc B, 2004, 66(2): 369-393

10 Jongbloed G, Van Der Meulen F H, Van Der Vaart A W. Non-parametric inference for Lévy driven Ornstein-Uhlenbeck processes. Bernoulli, 2005, 11(5): 759-791

11 Protter P. Stochastic Integration and Differential Equations. New York: Springer, 1990

12 Sato K. Lévy Processes and Infinitely Divisible Distributions. Cambridge: Cambridge University Press, 1999

13 Alexander I S, Wojbor A W. Distributions in the Physical and Engineering Sciences(Volume 1). Boston: Birkhäuser, 1997

14 Gaver D P Jr. Observing stochastic processes and approximate transform inversion. Oper Res, 1966, 14: 444-459 
15 Abate J, Whitt W. The Fourier-series method for inverting transforms of probability distributions. Queueing Systems, 1992, 10: 5-88

16 Abate J. Numerical inversion of Laplace transforms of probability distributions. ORSA Journal on Computing, 1995, 7(1): 36-43

17 Stehfest H, Algorithm 368. Numerical inversion of Laplace transforms. Commun ACM, 1970, 13: 47-49

18 Kallenberg O. Foundations of Modern Probability, Springer-Verlag, 1997

19 匡继昌. 常用不等式. 第三版. 济南: 山东科学技术出版社出版, 2004

20 Aitchison J, Silvey S D. Maximum-likelihood estimation of parameters subject to restraints. Ann Math Statist, 1958, 29: 813-828

21 Billingsley P. Statistical Inference for Markov Processes, Chicago, IL: The University of Chicago Press, 1961

22 Zhu R. On continuous-time generalized AR(1) processes models, statistical inference, and applications to non-normal time series. Dissertation for the Doctoral Degree. Vancouver: The University of British Columbia, 2002. 369-373 